\title{
The Numerical Solution of Boundary Value Problems for Stiff Differential Equations
}

\author{
By Joseph E. Flaherty* and R. E. O'Malley, Jr.**
}

\begin{abstract}
The numerical solution of boundary value problems for certain stiff ordinary differential equations is studied. The methods developed use singular perturbation theory to construct approximate numerical solutions which are valid asymptotically; hence, they have the desirable feature of becoming more accurate as the equations become stiffer. Several numerical examples are presented which demonstrate the effectiveness of these methods.
\end{abstract}

1. Introduction. Stiff initial and boundary value problems for ordinary differential equations arise in fluid mechanics, elasticity, electrical networks, chemical reactions, and many other areas of physical importance. Singularly perturbed problems, which are characterized by differential equations where the highest derivatives are multiplied by small parameters, are an important subclass of stiff problems. These problems must often be solved numerically; however, because they typically feature boundary layers (narrow intervals where the solution varies rapidly) their accurate numerical solution has been far from trivial.

Several schemes have been developed for the numerical solution of stiff initial value problems for ordinary differential equations; among them we note Gear's method [14] and the method of Bulirsch and Stoer [4]. These schemes work well for moderately stiff systems; however, as the stiffness increases they all require the use of very small mesh spacings over portions of the domain of integration. Thus, computational cost increases and accuracy decreases as the stiffness increases. Numerical schemes for two-point boundary value problems are not nearly as plentiful. This becomes clear when referring to the recent papers in Willoughby [44]. However, we note the work of Dorr [8] , [9] , Abrahamsson, Keller and Kreiss [1], Yarmish [45], and Ferguson [10]. Adaptive grid finite-difference schemes have been developed by Pearson [36], [37] for second order equations and by Keller [17] , [18] and Keller and White [20] for systems of first order equations. Keller's schemes have also been applied to partial differential equations [19]. Both Pearson's method and Keller's method use imbedding techniques to construct finite-difference grids which are dense within boundary layers; hence, while both techniques have been successfully applied to several problems, they require considerable computational effort for very stiff problems.

Received April 28, 1975; revised December 15, 1975.

AMS (MOS) subject classifications (1970). Primary 65L10, 34E15.

* The work of this author was supported in part by the National Science Foundation, Grant Number GP-27368.

** The work of this author was supported in part by the Office of Naval Research, Contract Number N0014-67 A-0209-0022.

Partial support was also provided by the Air Force Office of Scientific Research, Grant Number AFOSR-75-2818. 
We have developed algorithms which numerically construct asymptotic solutions of ordinary differential equations belonging to either a class of linear equations or quasilinear second order equations. In essence our methods use singular perturbation theory to construct the leading terms in formal asymptotic expansions of the solution. We solve for these leading terms using standard numerical techniques. We recall that classical singular perturbation methods (cf. Cole [5] or O'Malley [29]) separately solve a reduced (or outer) problem away from boundary layers and add appropriate solutions of boundary layer (or inner) problems where nonuniform convergence occurs. The outer solution follows from a regular perturbation (nonstiff) procedure, as do the inner solutions (although on a semi-infinite interval in the appropriate stretched boundary layer variable). Our numerical procedures avoid difficult stiff integrations in analogous fashion. While our methods are based principally on the work of O'Malley [32], similar ideas have also been used by Miranker [25], Aiken and Lapidus [2], Murphy [28], and MacMillan [23] for initial value problems. Their methods, like ours, have the important advantage of becoming more accurate as the equations become stiffer. This is because our solutions will be asymptotically valid as the small leading coefficients of the differential equation tend to zero.

Many important physical phenomena result in problems featuring nonuniformities away from the boundary points. Such interior nonuniformities can occur only when a turning point is encountered. This study does not consider these interesting, but more difficult problems, as it avoids solutions with turning points. Among numerical studies considering such possibilities we mention Dorr [9] , Pearson [36], Abrahamsson, Keller, and Kreiss [1], and Miranker and Morreeuw [26]. Somewhat related difficulties with stiff equation routines are the subject of Lindberg [22].

Our most complete results are for linear boundary value problems (Section 2); however, we also present results for some second order quasilinear problems (Section 3). We hope to later use Howes' recent study [16] to develop numerical algorithms for nonlinear boundary value problems with turning points. Several examples comparing our results with exact solutions, when known, and numerical solutions obtained by either a shooting procedure or by Pearson's method [36] are presented in Section 4. These results show that our methods can obtain accurate solutions to very stiff problems with very little computational effort.

2. Numerical Method for Linear Boundary Value Problems. We consider a linear ordinary differential equation of the form

$$
\sum_{j=0}^{m} a_{j}(x) \frac{d^{j} y}{d x^{j}}=0
$$

on the bounded interval $0 \leqslant x \leqslant 1$ subject to the linear boundary conditions

$$
y^{\left(\lambda_{i}\right)}(0)+\sum_{j=0}^{\lambda_{i}-1} b_{i j} y^{(j)}(0)=f_{i} ; \quad i=1,2, \ldots, r
$$

$$
m>\lambda_{1}>\lambda_{2}>\cdots>\lambda_{r}
$$




$$
\begin{aligned}
y^{\left(\lambda_{i}\right)}(1)+\sum_{j=0}^{\lambda_{i}-1} b_{i j} y^{(j)}(1)=f_{i} ; \quad i= & +1, r+2, \ldots, m, \\
m & >\lambda_{r+1}>\lambda_{r+2}>\cdots>\lambda_{m} .
\end{aligned}
$$

We assume that the leading coefficient $a_{m}(x)$ is small, but nonzero, throughout $0 \leqslant x$ $\leqslant 1$. Furthermore, the coefficients $a_{m-1}(x), a_{m-2}(x), \ldots, a_{n+1}(x)$ are (like $a_{m}$ ) small throughout $0 \leqslant x \leqslant 1$, but, $a_{n}(x)$ is not small on $0 \leqslant x \leqslant 1$. No specific dependence on a small parameter is assumed. We also assume that the boundary conditions have bounded coefficients $b_{i j}$ and $f_{i}$ which may be small.

We call the lower order differential equation

$$
\sum_{j=0}^{n} a_{j}(x) y^{(j)}(x)=0
$$

obtained by neglecting the small leading coefficients in (2.1), the reduced differential equation. Since $a_{n}(x)$ is nonzero, the reduced equation will be nonsingular, and the full equation (2.1) will not have turning points. (We refer the reader to Wasow [43], McHugh [24], or Olver [34] for a general discussion of turning point theory.)

Asymptotic solutions of $(2.1)$ are determined by the roots $\tilde{p}_{i}(x), i=1, \ldots, m$, of the characteristic polynomial

$$
\sum_{j=0}^{m} a_{j}(x) p^{j}(x)=0
$$

which has $m-n$ large roots determined asymptotically by the roots of the lower order polynomial

$$
\sum_{j=0}^{m-n} a_{n+j}(x) p^{j}(x)=0 .
$$

We assume that the roots $p_{i}(x), i=1,2, \ldots, m-n$, of $(2.5)$ all have nonzero real parts and are distinct throughout $0 \leqslant x \leqslant 1$. Furthermore, suppose that they are ordered so that

$$
\begin{cases}\operatorname{Re}\left[p_{i}(x)\right]<0, & i=1,2, \ldots, \sigma \\ \operatorname{Re}\left[p_{i}(x)\right]>0, & i=\sigma+1, \sigma+2, \ldots, \sigma+\tau=m-n\end{cases}
$$

Under the above assumptions O'Malley proves [32]:

THEOREM. (i) If the reduced problem

$$
\begin{cases}\sum_{j=0}^{n} a_{j}(x) y^{(j)}(x)=0, & \\ y^{\left(\lambda_{i}\right)}(0)+\sum_{j=0}^{\lambda_{i}-1} b_{i j} y^{(j)}(0)=f_{i} ; & i=\sigma+1, \ldots, r, \\ y^{\left(\lambda_{i}\right)}(1)+\sum_{j=0}^{\lambda_{i}-1} b_{i j} y^{(j)}(1)=f_{i} ; & i=r+\tau+1, \ldots, m,\end{cases}
$$

has a unique solution $z(x)$ and, (ii) if the two matrices 


$$
S=\left[\left(\frac{p_{j}(0)}{\max _{1 \leqslant k \leqslant \sigma}\left|p_{k}(0)\right|}\right)^{\lambda_{i}-\lambda_{\sigma}}\right], \quad i, j=1,2, \ldots, \sigma,
$$

and

$$
T=\left[\left(\frac{p_{\sigma+j}(1)}{\max _{1 \leqslant k \leqslant \tau}\left|p_{\sigma+k}(1)\right|}\right)^{\lambda_{r+i}-\lambda_{r+\tau}}\right], \quad i, j=1,2, \ldots, \tau,
$$

are both nonsingular, then there is a unique solution of the problem (2.1), (2.2) having the form

$$
y(x)=\widetilde{L}(x)+\widetilde{z}(x)+\widetilde{R}(x)
$$

where

$$
\widetilde{L}(x)=\sum_{i=1}^{\sigma} \frac{\widetilde{c}_{i} A_{i}(x) \exp \left[\int_{0}^{x} \tilde{p}_{i}(s) d s\right]}{\max _{1 \leqslant k \leqslant \sigma}\left|p_{k}(0)\right|^{\lambda_{\sigma}}}
$$

and

$$
\widetilde{R}(x)=\sum_{i=\sigma+1}^{m-n} \frac{\widetilde{c}_{i} A_{i}(x) \exp \left[\int_{1}^{x} \tilde{p}_{i}(s) d s\right]}{\max _{1 \leqslant k \leqslant \tau}\left|p_{\sigma+k}(1)\right|^{\lambda_{r}+\tau}} .
$$

The $A_{i}(x)$ are bounded and are chosen so that $A_{i}(0)=1$ for $i \leqslant \sigma$ and $A_{i}(1)=1$ otherwise. Furthermore, $\widetilde{z}(x)$ and $y(x)$ tend asymptotically to $z(x)$ within $0<x<1$.

We note that the boundary conditions for the reduced problem (2.7) are obtained by cancelling the first $\sigma$ boundary conditions (2.2a) at $x=0$ and the first $\tau$ boundary conditions (2.2b) at $x=1$. Thus, the signs of the large roots of the characteristic polynomial (2.4) are critical in defining the reduced problem. In particular, the reduced problem would not be defined if there were fewer than $\sigma$ boundary conditions at $x=0$ or fewer than $\tau$ boundary conditions at $x=1$. Indeed a limiting solution will not generally exist in such cases. Several additional consequences of the theorem are discussed by O'Malley [32], based on earlier work by Wasow [41] and O'Malley and Keller [33].

If the coefficients in (2.1) are sufficiently differentiable, then (2.10) can be differentiated repeatedly and provides an asymptotic representation of the derivatives of the solution. This can be used, as follows, to obtain more specific results about the solution $y(x)$ itself:

COROLlARY. Under the hypotheses of the theorem, the asymptotic solution of the problem (2.1), (2.2) satisfies

$$
y(x) \rightarrow L(x)+z(x)+R(x)
$$

uniformly within $0 \leqslant x \leqslant 1$. Here $L(x)$ is asymptotically zero unless $\lambda_{\sigma}=0$ and the left boundary layer jump $J_{L}=f_{\sigma}-z(0)$ is nonzero. Then, however,

$$
L(x) \sim \sum_{i=1}^{\sigma} c_{i} e^{p_{i}(0) x},
$$

where the $c_{i}$ are uniquely determined by the linear system 


$$
S\left[\begin{array}{l}
c_{1} \\
c_{2} \\
\cdot \\
\cdot \\
c_{\sigma}
\end{array}\right]=\left[\begin{array}{l}
0 \\
\cdot \\
\cdot \\
\cdot \\
0 \\
J_{L}
\end{array}\right] .
$$

Similarly, $R(x)$ is asymptotically zero unless $\lambda_{r+\tau}=0$ and the right boundary layer jump $J_{R}=f_{r+\tau}-z(1)$ is nonzero. Then

$$
R(x) \sim \sum_{i=\sigma+1}^{m-n} c_{i} e^{-p_{i}(1)(1-x)}
$$

where

$$
T\left[\begin{array}{l}
c_{\sigma+1} \\
c_{\sigma+2} \\
\cdot \\
\cdot \\
c_{m-n}
\end{array}\right]=\left[\begin{array}{l}
0 \\
\cdot \\
\cdot \\
\cdot \\
0 \\
J_{R}
\end{array}\right] .
$$

In particular, $L(x)$ and $R(x)$ are asymptotically zero within $0<x<1$.

Proof. Using (2.11a), we see that the left boundary layer correction is asymptotically negligible unless $\lambda_{\sigma}=0$, i.e., the last boundary condition cancelled at $x=0$ has the form $y(0)=f_{\sigma}$. When $\lambda_{\sigma}=0$, a boundary layer will generally be required at $x=0$, otherwise $y(x)$ will converge uniformly there, although its derivatives will not do so. Likewise, using $(2.11 \mathrm{~b})$, we see that $\widetilde{R}(x)$ is asymptotically negligible unless $\lambda_{r+\tau}=0$, when we generally get a boundary layer at $x=1$.

If $\lambda_{\sigma}=0$, the boundary layer behavior at $x=0$ can easily be asymptotically determined by finding limiting values for the constants $\tilde{c}_{i}$ of (2.11a). To this end, we substitute (2.10), (2.11) into (2.2a) and make use of the largeness of $\tilde{p}_{1}, \ldots, \tilde{p}_{\sigma}$ to obtain

$$
\begin{aligned}
& \sum_{i=1}^{\sigma} \frac{\widetilde{c}_{i}\left(p_{i}(0)\right)^{\lambda_{j}}}{\max _{1 \leqslant k \leqslant \sigma}\left|p_{k}(0)\right|^{\lambda_{\sigma}}}+\sum_{l=1}^{\lambda_{j}-1} b_{j l} \frac{\widetilde{c}_{i}\left(p_{i}(0)\right)^{l}}{\max _{1 \leqslant k \leqslant \sigma}\left|p_{k}(0)\right|^{\lambda_{\sigma}}} \\
& \quad+\left\{\widetilde{z}^{\left(\lambda_{j}\right)}(0)+\sum_{l=0}^{\lambda_{j}-1} b_{j l} \widetilde{z}^{(l)}(0)\right\} \sim f_{j}, \quad j=1, \ldots, \sigma .
\end{aligned}
$$

Because $\lambda_{\sigma}=0$ and $\lambda_{j}>\lambda_{\sigma}$ for $j<\sigma$ we asymptotically obtain (2.14), where $\tilde{c}_{i} \sim$ $c_{i}$ for $i=1, \ldots, \sigma$. By hypothesis (ii) of the theorem, (2.14) has a unique solution, which is trivial if $J_{L}=0$. Similar arguments at $x=1$ lead to (2.16).

Algorithm. We have used the above theorem and corollary to construct the following algorithm which yields approximate numerical solutions of the boundary value problem (2.1), (2.2).

(i) Determine the reduced equation (2.3) by sampling the coefficients in (2.1) for several values of $x$ on $[0,1]$. Also check that the leading coefficient $a_{m}(x)$ of 
the differential equation and the leading coefficient $a_{n}(x)$ of the reduced differential equation are nonzero on $[0,1]$.

(ii) Find the roots $p_{j}(x) ; j=1, \ldots, m-n$, of (2.5) using Muller's method [27] with the distinct initial guesses $\left[-a_{n}(0) / a_{m}(0)\right]^{1 /(m-n)}$. Check that the $p_{j}(x)$ all have nonzero real parts and are distinct throughout $[0,1]$. Calculate $\sigma$ and $\tau$ as defined in (2.6), and, hence, determine the boundary conditions for the reduced problem.

(iii) Calculate the matrices $S$ and $T$ defined in (2.8), (2.9) and check that they are nonsingular by using Gaussian Elimination to evaluate their determinants.

(iv) Solve the reduced problem by numerically finding a fundamental set of solutions of the reduced equation (2.7), i.e., by finding numerical solutions $z_{k}(x), k=1$, $2, \ldots, n$, of

$$
\begin{gathered}
\sum_{j=0}^{n} a_{j}(x) y^{(j)}(x)=0, \\
y^{(i)}(0)=\delta_{k-1, i}, \quad i=0,1, \ldots, n-1,
\end{gathered}
$$

where $\delta_{k, i}$ is the Kronecker delta. The solutions $z_{k}(x)$ are found on $0 \leqslant x \leqslant 1$ using a fourth order implicit Adam's method (cf. Gear [14]). The step size $h$ for the numerical integration is selected so that the maximum local discretization error $\tau(h)$ is such that

$$
\frac{\tau(h)}{\max _{0 \leqslant x \leqslant 1}\left|z_{k}(x)\right|} \approx \max \left[10^{-6}, \max _{0 \leqslant x \leqslant 1} \frac{\max _{n+1 \leqslant l \leqslant m}\left|a_{l}(x)\right|}{\max _{0 \leqslant l \leqslant n}\left|a_{l}(x)\right|}\right] .
$$

This insures that a solution with more accuracy than necessary is not obtained. The solution of the reduced problem is calculated as

$$
z(x)=\sum_{k=1}^{n} \alpha_{k} z_{k}(x),
$$

where the $\alpha_{k}$ are the solutions of the linear algebraic system

$$
\begin{gathered}
\alpha_{\lambda_{i}+1}+\sum_{j=0}^{\lambda_{i}-1} b_{i j} \alpha_{j+1}=f_{i}, \quad i=\sigma+1, \ldots, r, \\
\sum_{k=1}^{n}\left[z_{k}^{\left(\lambda_{i}\right)}(1)+\sum_{j=0}^{\lambda_{i}-1} b_{i j} z_{k}^{(j)}(1)\right] \alpha_{k}=f_{i}, \quad i=r+\tau+1, \ldots, m .
\end{gathered}
$$

If this system is nonsingular, then the reduced problem will have a unique solution. We note that the reduced problem is not stiff, hence, the reduced problem is solved without using a small step size.

(v) Add any boundary layer corrections to the reduced solution using (2.13), (2.14) and (2.15), (2.16). The matrices $S$ and $T$ are saved from step (iii) in factored form so that (2.14) and (2.16) are easily solved by forward and backward substitution.

We note that our procedure obtains a numerical approximation to the ledading terms of the appropriate asymptotic expansion of the solution without explicitly identifying the small parameters involved. This approach has also been used by several 
physicists and mathematicians (cf. Froman and Froman [13]). The asymptotic error of our approximation is uniformly $O(\epsilon)$, where we may take $\epsilon$ as either

$$
\epsilon=\max _{0 \leqslant x \leqslant 1 ; n+1 \leqslant j \leqslant m}\left|\frac{a_{j}(x)}{a_{n}(x)}\right|^{1 /(m-n)}
$$

or

$$
\epsilon=\min _{0 \leqslant x \leqslant 1 ; 1 \leqslant i \leqslant m-n} 1 /\left|p_{i}(x)\right| .
$$

This would be comparable to a relatively high order $O\left(h^{P}\right)$ of discretization error resulting from integration with a moderate step size $h$. Our results could be strengthened, if necessary, to a higher order asymptotic validity in $\epsilon$.

Our algorithm has been successfully applied to several examples which are discussed in Section 4.

3. A Method for Some Second Order Quasilinear Equations. To develop a general theory for analogous nonlinear boundary value problems would seem very difficult (cf. O'Malley [29] for some of the several complications that can occur). We note, in particular, that nonlinear initial value problems are far more tractable than two-point problems, which generally require growth restrictions of Nagumo-type on the nonlinearities. Except for the linear problems already discussed, we shall not treat initial value problems. We note, however, that methods based on such initial value problem algorithms have successfully been applied to classical optimization problems by Boggs [3] .

Our objective herein is to study only quasilinear scalar equations, although we anticipate that our ideas have wider applicability. Hence, we confine our attention to the boundary value problem

$$
\begin{gathered}
a_{0}(x, y) y^{\prime \prime}+a_{1}(x, y) y^{\prime}+a_{2}(x, y)=0, \quad 0 \leqslant x \leqslant 1, \\
y(0)=f_{1}, \quad y(1)=f_{2},
\end{gathered}
$$

where the $a_{i}$ 's are bounded for $y$ bounded and $a_{0}$ is small, but nonzero throughout $0 \leqslant x \leqslant 1$. We further restrict our attention to the two special cases when either (i) $a_{1} \equiv 0$ or (ii) $\left|a_{1} / a_{0}\right|$ is nowhere small compared to $\left|a_{2} / a_{0}\right|$.

Singular perturbation problems with $a_{0}(x, y)=\epsilon^{2}$ have been considered for both cases (i) and (ii) by O'Malley [29] and for case (i) by Fife [11], [12]. In particular, we recall Fife's result for the equation

$$
\epsilon^{2} y^{\prime \prime}=g(x, y), \quad 0 \leqslant x \leqslant 1,
$$

subject to the boundary conditions (3.2). He obtains a limiting solution $z(x)$ satisfying the reduced equation

$$
g(x, z(x))=0
$$

within $(0,1)$ provided that $g_{y}>0$ along $z(x)$ and

$$
\int_{z(0)}^{u} g(0, y) d y>0 \quad \text { for } u \text { between } z(0) \text { and (including) } f_{1}
$$


and

$$
\int_{z(1)}^{u} g(1, y) d y>0 \text { for } u \text { between } z(1) \text { and (including) } f_{2} \text {. }
$$

There are boundary layers at both endpoints. These conditions are weaker than more familiar ones requiring that $g_{y}$ remain negative in the boundary layers.

Analogously, O'Malley [29] has shown that the equation

$$
\epsilon^{2} y^{\prime \prime}+f(x, y) y^{\prime}+g(x, y)=0
$$

subject to boundary conditions (3.2) has a limiting solution $z(x)$ satisfying the terminal value problem

$$
f(x, y) y^{\prime}+g(x, y)=0, \quad y(1)=f_{2}
$$

within $(0,1]$, provided that, for some $\kappa>0, f \geqslant \kappa$ along $z(x)$ and

$$
\left(f_{1}-z(0)\right) \int_{z(0)}^{u} f(0, y) d y>0 \text { for } u \text { between } z(0) \text { and } f_{1} \text {. }
$$

There is then a boundary layer at $x=0$. If instead $f(x, y)$ were negative, the limiting solution would satisfy the boundary condition at $x=0$; and there would be a boundary layer at $x=1$ provided that the appropriate integral inequality is satisfied there.

Thus, for the boundary value problem (3.1), (3.2) without explicit dependence on a parameter, we may expect any bounded limiting solution $z(x)$ to satisfy either the reduced algebraic equation

$$
a_{2}(x, y)=0
$$

in case (i) or the reduced differential equation

$$
a_{1}(x, y) y^{\prime}+a_{2}(x, y)=0
$$

in case (ii): Many of the potential limiting solutions can be rejected as being inappropriate. However, our requirements are more stringent than necessary, so that we may eliminate some potentially valid limiting solutions. For example, because of their inherent difficulties, we avoid turning points by requiring that $a_{2 y}(x, z(x)) \neq 0$ when (3.9) applies and $a_{1}(x, z(x)) \neq 0$ when (3.10) applies.

Applying Fife's results, we obtain:

THEOREM. Consider the boundary value problem

$$
a_{0}(x, y) y^{\prime \prime}+a_{2}(x, y)=0, \quad y(0)=f_{1}, \quad y(1)=f_{2} .
$$

Suppose $z(x)$ satisfies the reduced problem

$$
a_{2}(x, z)=0
$$

and further satisfies

$$
\left\{\begin{array}{l}
a_{0}(x, z)<<a_{2 y}(x, z), \text { and } \\
\left.\frac{\partial}{\partial y}\left(\frac{a_{2}(x, y)}{a_{0}(x, y)}\right)\right|_{(x, z(x))}<0
\end{array}\right.
$$


throughout $0 \leqslant x \leqslant 1$ and that

$$
\left\{\begin{array}{l}
\frac{v_{L}(u)}{u} \equiv-\frac{1}{u} \int_{0}^{u} \frac{a_{2}(0, \bar{u}+z(0))}{a_{0}(0, \bar{u}+z(0))} d \bar{u}>>0, \text { and } \\
\frac{v_{R}(u)}{u} \equiv-\frac{1}{u} \int_{0}^{u} \frac{a_{2}(1, \bar{u}+z(1))}{a_{0}(1, \bar{u}+z(1))} d \bar{u}>>0
\end{array}\right.
$$

for $u$ between 0 and including $L(0) \equiv f_{1}-z(0)$ and $R(1) \equiv f_{2}-z(1)$, respectively.

Then, there exists an asymptotic solution $y(x)$ to (3.11) such that

$$
y(x) \rightarrow L(x)+z(x)+R(x)
$$

uniformly within $0 \leqslant x \leqslant 1$. The boundary layer corrections $L$ and $R$ are asymptotically zero within $(0,1)$. Their inverse functions are given by

respectively, where

$$
\left\{\begin{array}{l}
x(L)=\int_{L(0)}^{L} d u / w_{L}(u), \text { and } \\
x(R)=1+\int_{R(1)}^{R} d u / w_{R}(u)
\end{array}\right.
$$

$$
\begin{aligned}
& w_{L}(u)=-\sqrt{2 v_{L}(u)} \operatorname{sgn}(L(0)), \text { and } \\
& w_{R}(u)=-\sqrt{2 v_{R}(u)} \operatorname{sgn}(R(1)) .
\end{aligned}
$$

We note that within $(0,1)$, the asymptotic theory involves an $O(\mu)$ error for

$$
\mu^{2}=\max _{0 \leqslant x \leqslant 1}\left|\frac{a_{0}(x, z(x))}{a_{2 y}(x, z(x))}\right| .
$$

Within each boundary layer, the appropriate boundary layer equation should be studied (cf. O'Malley [29]). Thus, at $x=0$ the boundary layer correction $L(x)$ should satisfy

$$
a_{0}(0, L(x)+z(0)) \frac{d^{2} L}{d x^{2}}+a_{2}(0, L(x)+z(0))=0,
$$

have the initial value $L(0)$, and decay to zero for $x>0$. The differential equation (3.19) can be formally justified by introducing the small parameter

$$
\epsilon^{2}=\max \left|u / v_{L}(u)\right|
$$

for $u$ between 0 and $L(0)$, and the corresponding stretched variable $\xi=x / \epsilon$. Multiplying (3.19) by $d L / d x$ and integrating yields the implicit solution (3.16).

We note that the conditions (3.14) are guaranteed by (3.13) provided that the boundary layer jumps $|L(0)|$ and $|R(0)|$ are small. We also note that there is considerable practical advantage in obtaining $x$ as a function of $L$ (or $R$ ), because $L$ (or $R$ ) varies much more rapidly than $x$ in the boundary layers. Indeed, Vishik and Lyusternik [40] already used such changes of dependent and independent variables to convert singular perturbation problems to regular ones.

Relying on O'Malley [29], we obtain:

THEOREM. Consider the boundary value problem 


$$
a_{0}(x, y) y^{\prime \prime}+a_{1}(x, y) y^{\prime}+a_{2}(x, y)=0, \quad y(0)=f_{1}, y(1)=f_{2} .
$$

(a) Suppose $z(x)$ satisfies the reduced problem

$$
a_{1}(x, z) z^{\prime}+a_{2}(x, z)=0, \quad z(1)=f_{2}
$$

and further satisfies

$$
\left\{\begin{array}{l}
\frac{a_{1}(x, z)}{a_{0}(x, z)}>>0, \text { and } \\
a_{2}(x, z)=O\left(a_{1}(x, z)\right)
\end{array}\right.
$$

throughout $0 \leqslant x \leqslant 1$ and

$$
\frac{W_{L}(u)}{u} \equiv-\frac{1}{u} \int_{0}^{u} \frac{a_{1}(0, \bar{u}+z(0))}{a_{0}(0, \bar{u}+z(0))} d \bar{u}<<0
$$

for $u$ between 0 and including $L(0) \equiv f_{1}-z(0)$. Then, there exists an asymptotic solution $y(x)$ to $(3.21)$ such that

$$
y(x) \rightarrow L(x)+z(x)
$$

uniformly within $0 \leqslant x \leqslant 1$. The boundary layer correction $L(x)$ is asymptotically negligible for $x>0$ and has the inverse function

$$
x(L)=\int_{L(0)}^{L} d u / W_{L}(u)
$$

(b) Suppose, instead, that $z(x)$ satisfies the reduced problem

$$
a_{1}(x, z) z^{\prime}+a_{2}(x, z)=0, \quad z(0)=f_{1}
$$

and that

$$
\left\{\begin{array}{l}
\frac{a_{1}(x, z)}{a_{0}(x, z)}<<0, \text { and } \\
a_{2}(x, z)=O\left(a_{1}(x, z)\right)
\end{array}\right.
$$

throughout $0 \leqslant x \leqslant 1$ and

$$
\frac{W_{R}(u)}{u} \equiv-\frac{1}{u} \int_{0}^{u} \frac{a_{1}(1, \bar{u}+z(1))}{a_{0}(1, \bar{u}+z(1))} d \bar{u}>>0
$$

for $u$ between 0 and including $R(1)=f_{2}-z(1)$. Then, there exists an asymptotic solution $y(x)$ to $(3.21)$ such that

$$
y(x) \rightarrow z(x)+R(x)
$$

uniformly within $0 \leqslant x \leqslant 1$. Here, the boundary layer correction $R(x)$ is asymptotically negligible for $x<1$ and has the inverse function

$$
x(R)=1+\int_{R(0)}^{R} d u / W_{R}(u)
$$


In these results, the asymptotic solution has an $O(\mu)$ error within $(0,1)$ for

$$
\mu=\max _{0 \leqslant x \leqslant 1}\left|\frac{a_{0}(x, z(x))}{a_{1}(x, z(x))}\right|,
$$

while the error of the boundary layer correction $L(x)$, for example, is $O(\epsilon)$ for

$$
\epsilon=\max \left|\frac{u}{w_{L}(u)}\right|
$$

with $u$ between 0 and $L(0)$. The appropriate boundary layer equation is

$$
a_{0}(0, L(x)+z(0)) \frac{d^{2} L}{d x^{2}}+a_{1}(0, L(x)+z(0)) \frac{d L}{d x}=0
$$

Its unique decaying solution for the given initial value is expressed in (3.26). If $z(0)=$ $f_{1}$, of course, there is no need for the boundary layer correction.

Several complications can occur for the equation (3.1) if $a_{1}$ is allowed to be small. If (3.1) has a limiting solution $z(x)$ satisfying $a_{2}(x, z(x))=0$, then $a_{0}$ and $a_{1}$ must be small along $z(x)$. Additional restrictions are required at the endpoints in order to allow for the necessary boundary layers. This is clear from the linear problem

$$
\epsilon y^{\prime \prime}+\mu a y^{\prime}+b y=0, \quad b \neq 0,
$$

where the solution depends critically on the size of $\epsilon / \mu^{2}$ as $\epsilon$ and $\mu$ both tend to zero (see Section 4). Moreover, the thickness of the boundary layer changes significantly if $a_{1}$ ceases to be small within the boundary layer. In fact solutions can fail to exist. O'Malley [32], for example, considered the equation

$$
\epsilon y^{\prime \prime}+a_{1}(y) y^{\prime}-y=0,
$$

where $a_{1}$ is a smooth, monotonically increasing function satisfying

$$
a_{1}(y)= \begin{cases}2, & 1 / 2 \leqslant y \leqslant 1, \\ 0, & 0 \leqslant y \leqslant 1 / 4 .\end{cases}
$$

For $y(0)=y(1)=1$, the problem has a solution tending to the solution of the reduced problem

$$
2 y^{\prime}-y=0, \quad y(1)=1
$$

for $x>0$ with a boundary layer of thickness $O(\epsilon)$. It was claimed that another solution tended to zero within $(0,1)$. This would be true for $y(0)=y(1)<1 / 4$, but not for the prescribed conditions. (This is because the boundary layer interval with $a_{1}>$ 0 corresponds to a boundary layer of thickness $O(\epsilon)$ at $x=0$, rather than the $O(\sqrt{\epsilon})$ boundary layers at both endpoints encountered when $a_{1} \equiv 0$.) Thus for nonlinear problems, convergence for $\epsilon \longrightarrow 0$ often requires restrictions on the size of the boundary layer jumps. Such restrictions have been required in the analytic theory (cf. Wasow [42]), but can sometimes be removed.

In both cases (i) and (ii) we have required that the boundary conditions prescribe $y$ at each endpoint. If the boundary condition, say at $x=0$, has the form 


$$
y^{\prime}(0)+b_{10} y(0)=f_{1},
$$

then rapid decay of the boundary layer correction will require $y^{\prime}(0)$ to be bounded and the boundary layer jump to be small to satisfy (3.39).

Algorithm. We have used the above theoretical results, as follows, to construct approximate numerical solutions of (3.1), (3.2):

(i) Find solutions of the reduced problem.

(a) In case (i) we use Muller's method [27] to find all real finite roots of $a_{2}(0, y)=0$. Then, using these roots as initial guesses, we solve (3.9) for $z(x)$ by Newton's method in steps of $h=1 / N$ from $x=0$ to 1 . We reject any solution that does not satisfy the sign condition on $\left(a_{2} / a_{0}\right)_{y}$.

(b) In case (ii) we use a fourth order Adam's predictor-corrector method to solve (3.10) subject to one of the boundary conditions (3.2). The step size $h$ is selected in the same manner as described for linear equations in Section 2 . We reject any solution that does not satisfy the sign condition on $a_{1} / a_{0}$.

(ii) Calculate $v_{L}(u)$ and $v_{R}(u)$ or $W_{L}(u)$ or $W_{R}(u)$ using the appropriate equation [either (3.14), (3.24), or (3.29)] by the Corrected Trapezoidal Rule [7] with $M$ uniform steps of either $h=\left(f_{1}-z(0)\right) / M$ or $h=\left(f_{2}-z(1)\right) / M$ for $u$ between 0 and either $f_{1}-z(0)$ or $f_{2}-z(1)$, respectively. We reject any solution which does not satisfy the appropriate sign condition on $v_{L}, v_{R}, W_{L}$ or $W_{R}$. The Corrected Trapezoidal Rule is used because of its accuracy and because the derivatives involved must be available to perform the sign test in (i)(a).

(iii) Calculate $x(L)$ and/or $x(R)$ from either (3.16), (3.26), or (3.31). The integrands involved in this calculation are all singular at $L$ or $R=0$, so, in order to preserve numerical accuracy, we evaluate them in a modified form. For example, we rewrite (3.16a) as

$$
x(L)=\ln \left(\frac{L}{f_{1}-z(0)}\right)+\int_{f_{1}-z(0)}^{L}\left[\frac{1}{w_{L}(u)}-\frac{1}{u}\right] d u .
$$

The modified integrands are evaluated by the Trapezoidal rule for $L=f_{1}-z(0)$ or $R=f_{2}-z(1)$ until either $|u|<10^{-7}$ or $x$ is outside the interval $[0,1]$. (We note that the hypotheses imposed imply that the boundary layer equations are asymptotically stable in the appropriate stretched variable.)

(iv) Add the boundary layer correction(s) to the reduced solution.

4. Numerical Examples. We have conducted several numerical experiments which compare the results of our asymptotic methods to exact solutions, when known, and numerical solutions obtained by either a shooting procedure or by Pearson's method [36], [37].

The shooting procedure has been coded to solve linear boundary value problems of the form (2.1), (2.2) using Gear's method [14] to construct a fundamental set of solutions to initial value problems for the equation and Gaussian Elimination to solve the linear algebraic system that determines the initial conditions. 
Pearson's method uses a variable mesh spacing on $[0,1]$ and approximates first and second derivatives at a mesh point $x_{j}$ by

$$
\begin{gathered}
y^{\prime}\left(x_{j}\right) \approx \frac{h_{j-1}^{2}\left(y_{j+1}-y_{j}\right)+h_{j}^{2}\left(y_{j}-y_{j-1}\right)}{h_{j} h_{j-1}\left(h_{j}+h_{j-1}\right)}, \\
y^{\prime \prime}\left(x_{j}\right) \approx \frac{h_{j-1}\left(y_{j+1}-y_{j}\right)-h_{j}\left(y_{j}-y_{j-1}\right)}{h_{j} h_{j-1}\left(h_{j}-h_{j-1}\right)},
\end{gathered}
$$

where $h_{j}=x_{j+1}-x_{j}$ and $y_{j}$ denotes the numerical approximation to $y\left(x_{j}\right)$. We use these approximations in either a second order linear differential equation or a second order quasilinear differential equation of the form (3.1). This gives either a linear or nonlinear tridiagonal algebraic system, respectively, which must be solved for the values of $y_{j}$ at each mesh point. For a given mesh spacing we solve the linear algebraic system directly using the tridiagonal algorithm, while we solve the nonlinear algebraic system by Newton iteration using the tridiagonal algorithm. For singularly perturbed differential equations this process can give eroneous results unless the mesh spacing is sufficiently dense within the boundary layers. This relates to the difference equation not being of positive type (cf. Parter [35] or Hemker [15]). Pearson's idea is to solve the problem in $\epsilon$-steps. (For differential equations without explicit dependence on a small parameter, we may take $\epsilon$ as the ratio of the maximum absolute value of the small coefficients, provided that the nonsmall coefficients are $O(1)$. Such an $\epsilon$ is generated by our procedure.) Thus, we solve a series of problems in which the parameter $\epsilon$ is made successively smaller. The mesh is first made uniform and, for quasilinear equations, we use the result of our asymptotic method as an initial guess for the $y_{j}$. After the appropriate algebraic system has been solved for $y_{j}$, the mesh spacing is adjusted by adding additional points between any pair of adjacent mesh points, say $x_{j}$ and $x_{j+1}$, where $\left|y_{j+1}-y_{j}\right|>\delta\left\{\max _{j}\left|y_{j}\right|-\min _{j}\left|y_{j}\right|\right\}$. We performed our calculations with $\delta=10^{-2}$ and $10^{-3}$ and used Pearson's [36] algorithms for adding mesh points and smoothing the new mesh. The algebraic system is resolved until no new points are added. The entire process is then repeated for a smaller value of $\epsilon$ using the mesh spacing and, for quasilinear equations, the previous step's $y_{j}$ values as initial guesses.

Both the shooting procedure and Pearson's method were chosen for our numerical study primarily because they are easily coded. They would in general not be competitive with more recently developed procedures such as Scott and Watts' orthogonalization code [38], Keller's finite-difference methods [17] , [18] , [20], or Lentini and Pereyra's deferred correction routines [21]. Nevertheless, the following comparisons clearly demonstrate the principal advantage of our methods, namely that they increase in accuracy, without additional computational cost, as the equation becomes stiffer. Thus, for those problems where our methods are applicable, they would eventually surpass, in both accuracy and speed, any method that requires additional computational effort as stiffness increases. 
Example la: $\quad \varepsilon=\mu$ 5/2

\begin{tabular}{|c|c|c|c|c|}
\hline$\mu$ & $\max _{x \in[0,1]} e_{A}(x)$ & $\max _{x \in[0,1]} e_{s}(x)$ & $\max _{x \in[0,1]}{ }^{e_{P 2}(x)}$ & $\max _{x \in[0,1]}{ }^{e_{P 3}(x)}$ \\
\hline $10^{-1}$ & $1.7(-4)$ & $4.6(-4)$ & $3.3(-4)$ & $3.2(-6)$ \\
\hline $10^{-2}$ & 0 & * & $7.4(-4)$ & $5.9(-6)$ \\
\hline $10^{-3}$ & 0 & & $9.4(-4)$ & $\star \star$ \\
\hline $10^{-4}$ & 0 & & $7.2(-4)$ & \\
\hline
\end{tabular}

\begin{tabular}{|c|c|c|c|c|}
\hline $10^{-1}$ & $1.0(-3)$ & $2.2(-7)$ & $1.0(-4)$ & $9.4(-7)$ \\
\hline $10^{-2}$ & $4.6(-8)$ & * & $1.7(-4)$ & $1.6(-6)$ \\
\hline $10^{-3}$ & $1.1(-7)$ & & $1.8(-4)$ & $1.7(-6)$ \\
\hline $10^{-4}$ & 0 & & $1.7(-4)$ & $\star \star$ \\
\hline
\end{tabular}

Example lc: $\varepsilon=\mu$

\begin{tabular}{l|l|l|l|l|}
\hline $10^{-1}$ & $3.4(-2)$ & $1.2(-7)$ & $1.8(-5)$ & $2.2(-7)$ \\
$10^{-2}$ & $3.7(-5)$ & $1.3(-6)$ & $5.0(-5)$ & $5.4(-7)$ \\
$10^{-3}$ & $4.9(-8)$ & $\star$ & $2.0(-5)$ & $6.9(-7)$ \\
$10^{-4}$ & $4.6(-8)$ & & $3.7(-5)$ & $1.7(-6)$ \\
\hline
\end{tabular}
$\begin{aligned} & \text { Norm. avg } \\
& \text { exec time }\end{aligned}$

TABLE 1

Comparison of exact and numerical solutions for Example 1. A* indicates that a solution could not be found using single precision arithmetic. $\mathrm{A}^{* *}$ indicates that a solution could not be found using less than 5002 points

In all of the tables describing the results of our numerical experiments we use the subscript $E$ to denote the exact solution, $A$ to denote our approximate numerical solution, $S$ to denote the solution obtained by shooting, $P 2$ to denote the solution obtained by Pearson's method with $\delta=10^{-2}$, and $P 3$ to denote the solution obtained by Pearson's method with $\delta=10^{-3}$. We compare the results of the numerical methods to the exact solution whenever the exact solution is known. We use the symbol $e$ to denote the absolute difference between the exact solution and a numerical solution; hence, $e_{A}(x)=\left|y_{E}(x)-y_{A}(x)\right|$. Whenever the exact solution is not known we compute the difference between the asymptotic solution and a numerical solution using the symbol $d$ to denote this difference. Thus, $d_{S}(x)$ denotes $\left|y_{S}(x)-y_{A}(x)\right|$. Differences recorded 
Example 2a: $\quad c=\mu^{5 / 2}$

\begin{tabular}{|c|c|c|c|c|}
\hline$\mu$ & $\begin{array}{c}\text { Interval } \\
I=\left[x_{L}, x_{R}\right]\end{array}$ & $\max _{x \in I}\left|Y_{E}(x)\right|$ & $\max _{x \in I} e_{A}(x)$ & $\begin{array}{l}\max e_{S}(x) \\
x \in I\end{array}$ \\
\hline $10^{-2}$ & $0,10^{-2}$ & 1.0 & $2.0(-9)$ & $6.5(-8)$ \\
$10^{-2}, 0.1$ & $0.1,1.0$ & $1.5(-5)$ & $3.8(-8)$ & $1.6(-7)$ \\
$10^{-3}$ & $0,10^{-2}$ & 1.0 & $4.3(-8)$ & $2.2(-9)$ \\
\hline & $10^{-2}, 1$ & $2.8(-5)$ & $2.3(-11)$ & $6.7(-7)$ \\
\hline
\end{tabular}

Example 2b: $\quad \varepsilon=\mu^{3 / 2}$

\begin{tabular}{|l|l|l|l|l|}
\hline $10^{-1}$ & $0,1.0$ & 1.0 & 0 & $2.0(-4)$ \\
\hline $10^{-2}$ & $0,1.0$ & 1.0 & 0 & $3.1(-4)$ \\
\hline $10^{-3}$ & $0,1.0$ & 1.0 & 0 & $6.8(-4)$ \\
\hline $10^{-4}$ & $0,1.0$ & 1.0 & 0 & $2.0(-3)$ \\
\hline
\end{tabular}

Norm. avg Exec time

TABLE 2

Comparison of exact and numerical solutions for Example 2

as 0 in the tables imply agreement to at least seven significant digits. We terminated any calculation using the shooting method when an answer could not be obtained with a minimum step size larger than $10^{-13}$ and single precision arithmetic. We terminated any calculation using Pearson's method when an answer could not be obtained with less than 5002 mesh points. All calculations were performed on a CDC 6600 computer at the Courant Institute, New York University.

We present the results of numerical experiments on five linear differential equations and three nonlinear differential equations.

Example 1. $\epsilon y^{\prime \prime}+\mu y^{\prime}-y=0, y(0)=1, y(1)=1 / 2$ where $\epsilon$ and $\mu$ are small constant parameters. This example was introduced by O'Malley [32] and is interesting because different boundary layer behaviors result depending on whether $\epsilon / \mu^{2} \rightarrow 0$, $\epsilon / \mu^{2} \rightarrow 1$, or $\epsilon / \mu^{2} \rightarrow \infty$ as $\mu \rightarrow 0$. In all cases the limiting solution within $(0,1)$ is trivial and the solution is easily determined asymptotically by expanding the exact solution of the constant coefficient equation. We made runs for $\epsilon=\mu^{5 / 2}$ (Example 1a), $\epsilon=\mu^{2}$ (Example 1b), and $\epsilon=\mu$ (Example 1c) for $\mu=10^{-i}, i=1,2,3,4$. Comparisons between the exact and numerical solutions are presented in Table 1. The average execution time per calculation performed, normalized with respect to the execution time of the asymptotic solution is also presented in the table. We observe that the shooting procedure does quite poorly for this example as it did for all examples with boundary layers at both ends of the interval. In all three cases our method yielded 


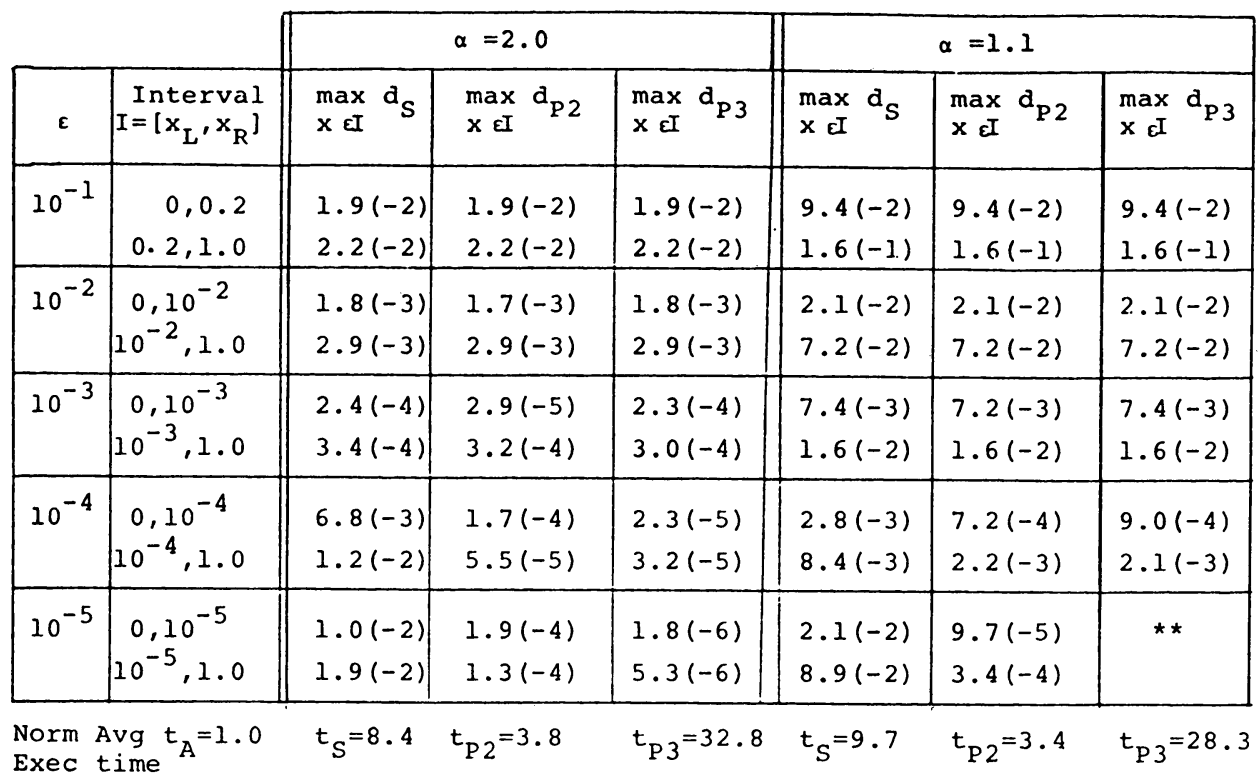

TABLE 3

Difference between asymptotic and numerical solutions for Example 3. $\mathbf{A}^{* *}$ indicates that a solution could not be found using less than 5002 points

\begin{tabular}{|l|c|c|}
\hline$\varepsilon$ & $\max _{x \in[0,1]}{ }^{e} A$ & $\begin{array}{c}\max \\
x \varepsilon[0,1]\end{array}{ }^{e} S$ \\
\hline $10^{-1}$ & $1.3(-1)$ & $1.1(-7)$ \\
\hline $10^{-2}$ & $8.2(-2)$ & $6.0(-6)$ \\
\hline $10^{-3}$ & $3.4(-2)$ & $\star$ \\
\hline $10^{-4}$ & $1.2(-2)$ & \\
\hline $10^{-5}$ & $4.0(-3)$ & \\
\hline
\end{tabular}

Norm avg Exec time $t_{A}=1.0$

$t_{S}=10.1$

TABLE 4

Comparison of exact and numerical solutions for Example 4. A* indicates that a solution could not be found using single precision arithmetic

very accurate results for $\mu \leqslant 10^{-2}$ for very little computational cost, and for Examples $1 \mathrm{a}$ and $1 \mathrm{~b}$, it also gave accurate results for $\mu=10^{-1}$. Graphs of the asymptotic solutions of Examples 1a, 1b, and 1c are presented in Figures 1, 2, and 3, respectively. The exact solution of Example 1c for $\mu=10^{-1}$ is also shown in Figure 3 where there are visible differences between the exact and asymptotic results. We note that Example 1a 


\begin{tabular}{|c|c|c|c|}
\hline$\varepsilon$ & $\begin{array}{r}\text { Interval } \\
I=\left[x_{L}, x_{R}\right]\end{array}$ & $\max _{x \in I}\left|y_{S}(x)\right|$ & $\max _{x \in I} d_{S}$ \\
\hline $10^{-2}$ & $\begin{array}{r}0,0.5 \\
0.5,1.0\end{array}$ & $\begin{array}{l}0.14 \\
1.0\end{array}$ & $\begin{array}{l}3.5(-1) \\
2.7(-1)\end{array}$ \\
\hline $10^{-4}$ & $\begin{array}{r}0,0.8 \\
0.8,1.0\end{array}$ & $\begin{array}{c}0.069 \\
1.0\end{array}$ & $\begin{array}{l}3.9(-2) \\
2.3(-2)\end{array}$ \\
\hline $10^{-6}$ & $\begin{array}{r}0,0.9 \\
0.9,1.0\end{array}$ & $\begin{array}{c}0.068 \\
1.0\end{array}$ & $\begin{array}{l}8.8(-3) \\
7.3(-3)\end{array}$ \\
\hline
\end{tabular}

TABLE 5

Differences between asymptotic and shooting solutions for Example 5

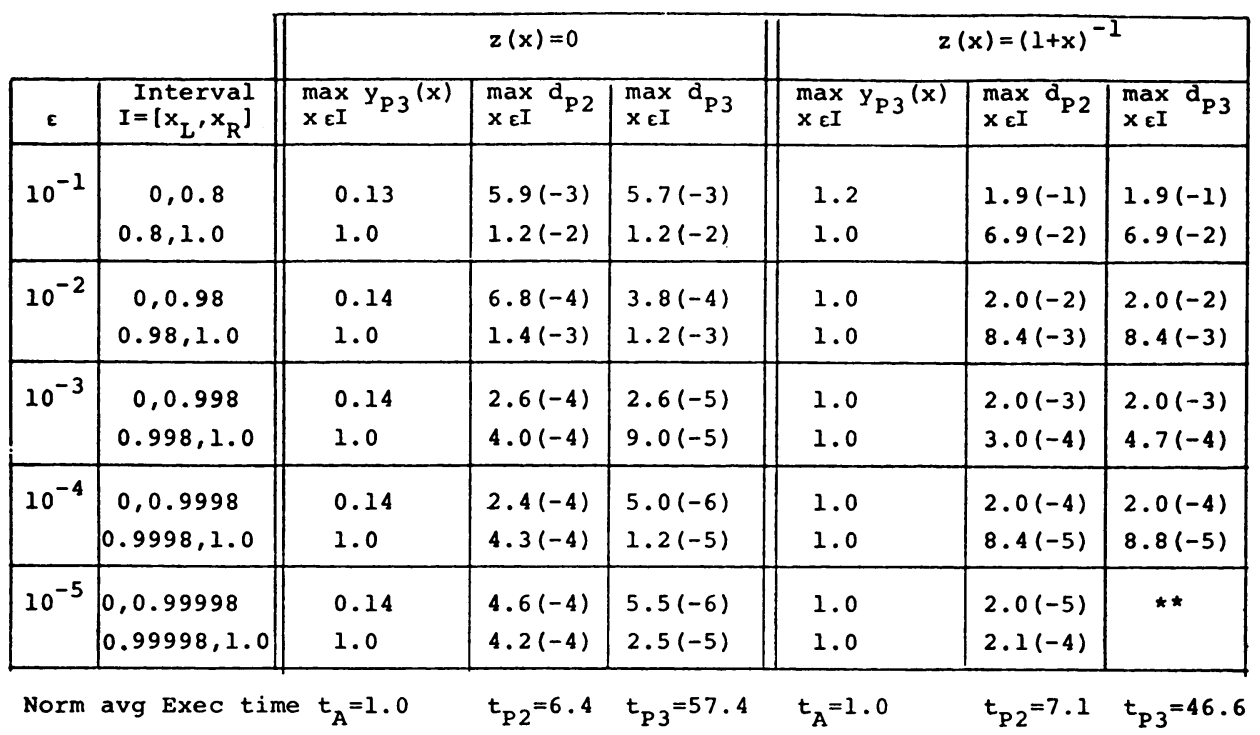

TABLE 6

Differences between asymptotic and Pearson's solutions for Example 6. $\mathrm{A}^{* *}$ indicates that a solution could not be obtained using less than 5002 points

has a boundary layer of order $O(\epsilon / \mu)=O\left(\mu^{3 / 2}\right)$ at $x=0$ and one of order $O(\mu)$ at $x=$ 1 while boundary layers are of order $O(\mu)$ and $O(\sqrt{\epsilon})=O(\sqrt{\mu})$ at both endpoints for Examples $1 \mathrm{~b}$ and $1 \mathrm{c}$, respectively. These differences are observed on the figures. In particular, observe that the boundary layer for Example 1c and $\mu=0.1$ is of order $O(\sqrt{.1})$, so we should not expect our asymptotic results to be accurate. They obviously improve with stiffness.

Example 2. $\epsilon y^{\prime \prime}+\mu y^{\prime}+y=0, y(0)=1, y^{\prime}(0)=0$. For $\epsilon$ and $\mu$ positive and tending to zero there are limiting solutions to the initial value problem but not to problems prescribing $y$ at each end. Two cases were considered: $\epsilon=\mu^{5 / 2}$ (Example 


\begin{tabular}{|c||c|c||c|c|}
\cline { 2 - 5 } \multicolumn{1}{|c||}{} & \multicolumn{2}{c||}{ Example 7 } & \multicolumn{2}{c|}{ Example 8 } \\
\hline$\varepsilon$ & $\max d_{P 2}$ & $\begin{array}{c}\max d_{P 3} \\
{[0,1]}\end{array}$ & $\begin{array}{c}\max d_{P 2} \\
{[0,1]}\end{array}$ & $\begin{array}{c}\max d_{P 3} \\
{[0,1]}\end{array}$ \\
\hline $10^{-1}$ & $6.7(-2)$ & $6.7(-2)$ & $4.8(-1)$ & $4.8(-1)$ \\
\hline $10^{-2}$ & $1.8(-4)$ & $1.8(-4)$ & $5.5(-5)$ & $5.5(-5)$ \\
\hline $10^{-3}$ & $2.5(-5)$ & $1.1(-5)$ & $2.4(-5)$ & $5.2(-6)$ \\
\hline $10^{-4}$ & $2.6(-5)$ & $\star \star$ & $3.4(-5)$ & $4.0(-6)$ \\
\hline $10^{-5}$ & $2.9(-5)$ & & $3.9(-5)$ & $\star \star$ \\
\hline
\end{tabular}

$\begin{array}{llll}\text { Norm Avg } & \text { example 7: } t_{A}=1.0 & t_{P 2}=9.6 & t_{P 3}=64.6 \\ \text { Exec time } & \text { example 8: } t_{A}=1.0 & t_{P 2}=5.9 & t_{P 3}=28.7\end{array}$

TABLE 7

Differences between asymptotic and numerical solutions for

Examples 7 and $8 . \mathrm{A}^{* *}$ indicates that a solution could not be obtained using less than 5002 points

2a) which has a monotonically decaying initial layer and $\epsilon=\mu^{3 / 2}$ (Example 2b) which has a damped oscillatory initial layer (see Figure 4). Runs were made for $\mu=10^{-i}$ with $i=2,3$ in Example 2a and $i=1,2,3,4$ in Example 2b and comparisons between exact, shooting, and asymptotic solutions are presented in Table 2. We note that our numerical results are superior to those obtained by Gear's method.

Example 3. $\epsilon y^{\prime \prime}+\left(\alpha-x^{2}\right) y^{\prime}-x y=0, y(0)=1, y(1)=1 / 2$. The results of runs for $\alpha=2.0$ and 1.1 with $\epsilon=10^{-i}, i=1, \ldots, 5$, are presented in Table 3. The effects of round off errors begin to dominate the solution obtained by shooting for $\epsilon \leqslant$ $10^{-4}$ and for $\epsilon \leqslant 10^{-5}$ accurate solutions obtained by Pearson's method require considerable computational effort. If $0 \leqslant \alpha \leqslant 1$, then the differential equation would have a turning point at $x=\sqrt{\alpha}$ and our methods would cease to be applicable. Hence, the differential equation with $\alpha=1.1$ is a tougher problem than the equation with $\alpha=$ 2.0. This explains why our results are not very accurate for $\epsilon \geqslant 10^{-3}$ with $\alpha=1.1$; however, as $\epsilon$ becomes smaller our results become increasingly more accurate. Graphs of the asymptotic solution are presented for $\alpha=2.0$ and 1.1 in Figures 5 and 6, respectively. When significant differences are apparent, the solution by Pearson's method is also shown.

Example 4. $\epsilon y^{\mathrm{iv}}-(1+\epsilon) y^{\prime \prime}+y=0, y(0)=y^{\prime}(0)=y^{\prime}(1)=0, y(1)=1$. This equation was introduced by Conte [6] and can, of course, be solved exactly. The solution of the reduced problem

$$
-(1+\epsilon) z^{\prime \prime}+z=0, \quad z(0)=0, \quad z(1)=1
$$

converges uniformly to $y$ on $[0,1]$ while the derivative $y^{\prime}$ has boundary layers of thickness $O(\sqrt{\epsilon})$ at each endpoint. Runs were made for $\epsilon=10^{-i}, i=1, \ldots, 5$, and the results of the asymptotic and shooting solutions are compared with the exact solution in Table 4. Graphs of the asymptotic and exact solutions are presented in Figure 7. We note, in particular, the difficulty encountered by the shooting solution for small values of $\epsilon$. 


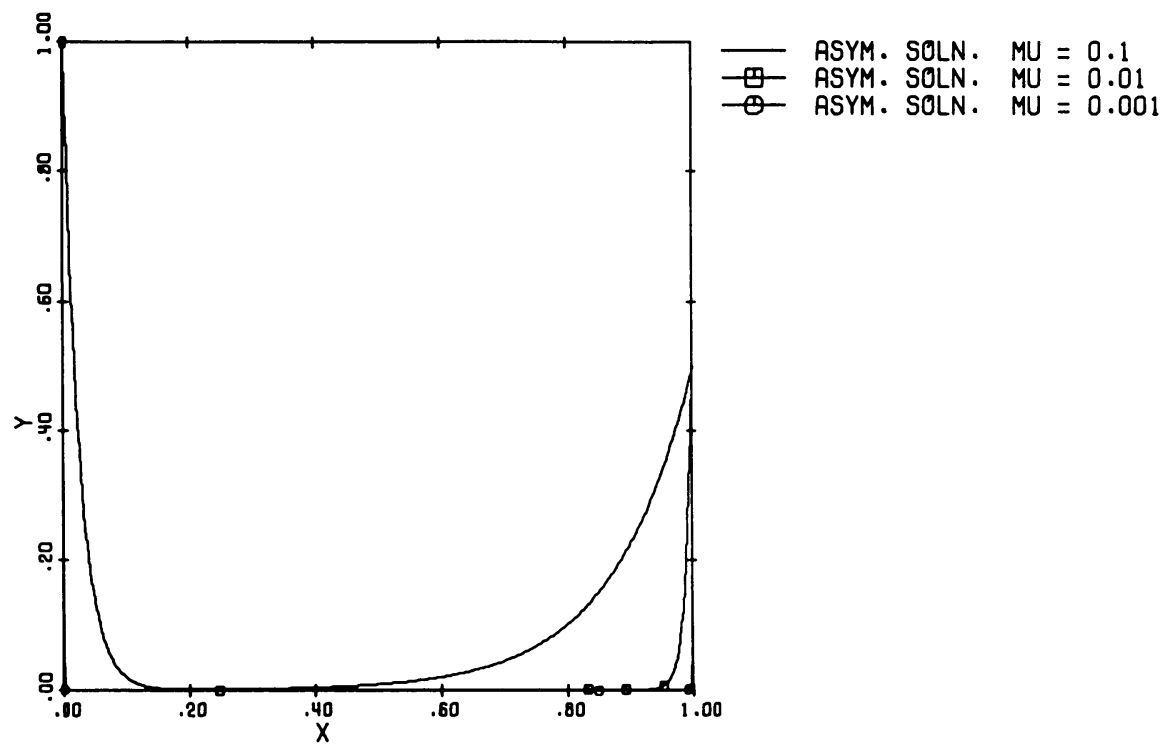

Figure 1

Asymptotic solution of Example 1a: $\epsilon y^{\prime \prime}+\mu y^{\prime}-y=0, y(0)=1$,

$$
y(1)=1 / 2, \epsilon=\mu^{5 / 2}
$$

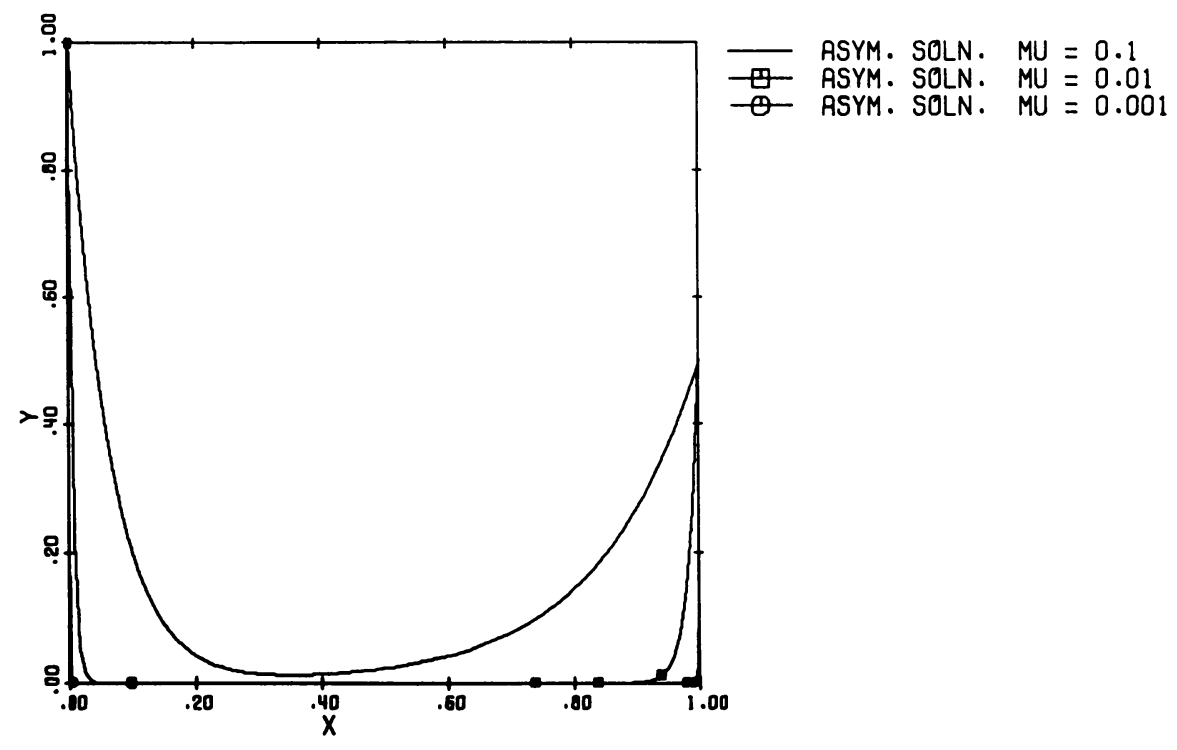

Figure 2

Asymptotic solution of Example $1 \mathrm{~b}: \epsilon y^{\prime \prime}+\mu y^{\prime}-y=0, y(0)=1$,

$$
y(1)=1 / 2, \epsilon=\mu^{2}
$$



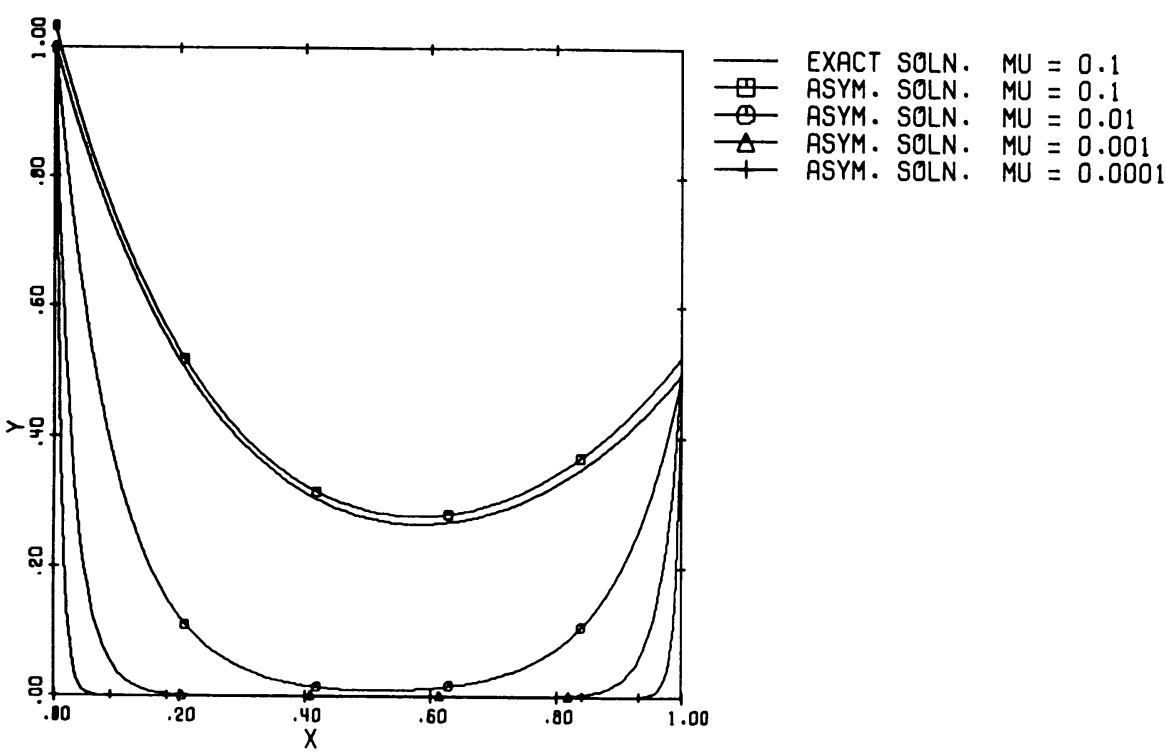

Figure 3

Exact and asymptotic solutions of Example 1c: $\epsilon y^{\prime \prime}+\mu y^{\prime}-y=0$,

$$
y(0)=1, y(1)=1 / 2, \epsilon=\mu
$$

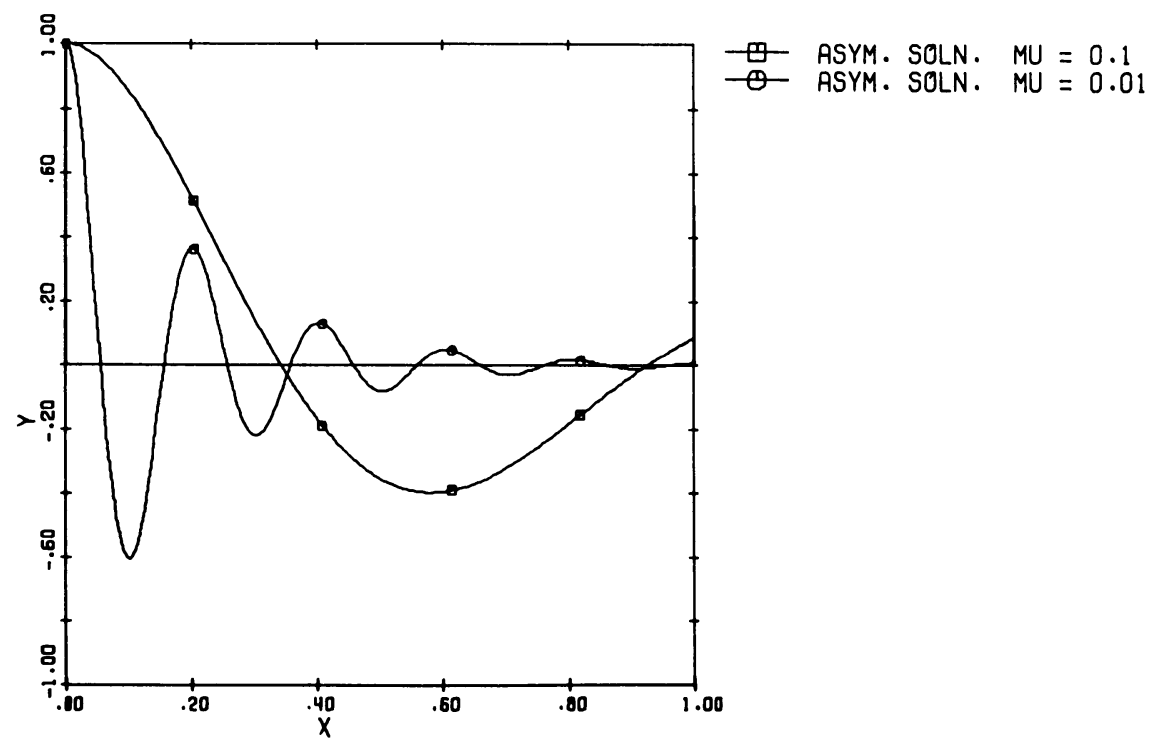

FIGURE 4

Asymptotic solution of Example $2 \mathrm{~b}: \epsilon y^{\prime \prime}+\mu y^{\prime}+y=0, y(0)=1$,

$$
y^{\prime}(0)=0, \epsilon=\mu^{3 / 2}
$$




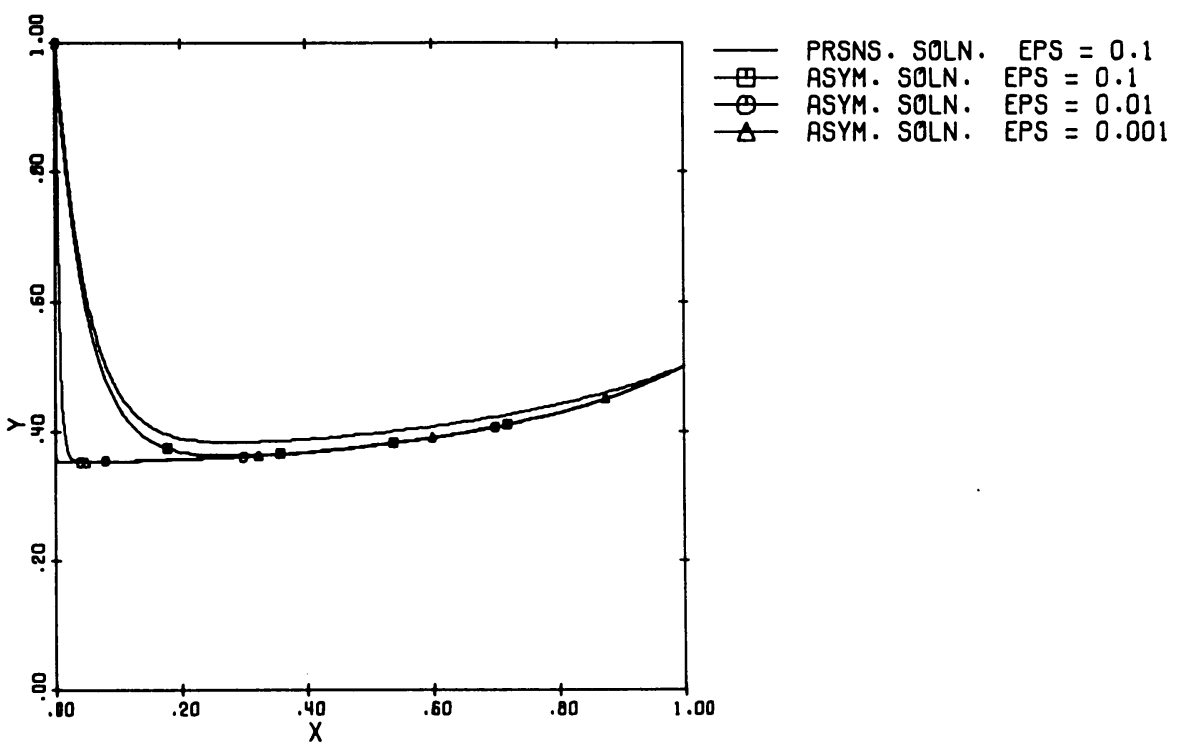

Figure 5

Asymptotic and Pearson's method solution of Example 3: $\epsilon y^{\prime \prime}+$ $\left(\alpha-x^{2}\right) y^{\prime}-x y=0, y(0)=1, y(1)=1 / 2, \alpha=2$

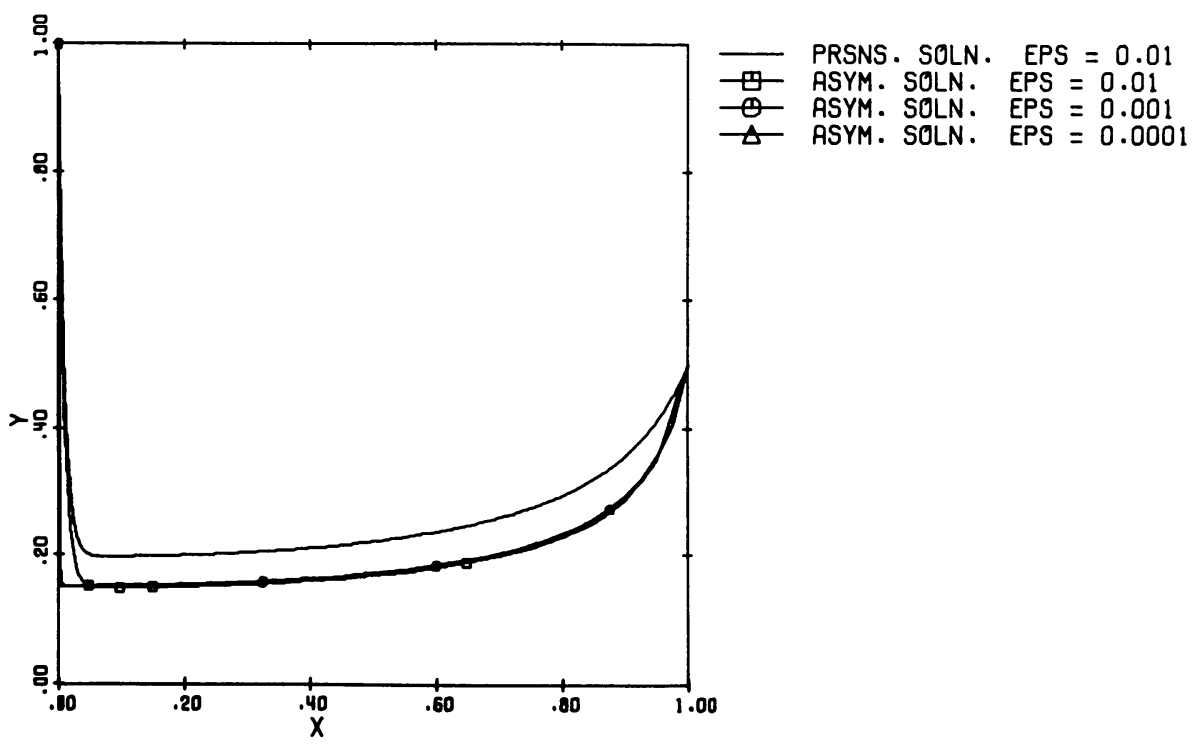

FIGURE 6

Asymptotic and Pearson's method solution of Example 3: $\epsilon y^{\prime \prime}+$ $\left(\alpha-x^{2}\right) y^{\prime}-x y=0, y(0)=1, y(1)=1 / 2, \alpha=1.1$ 


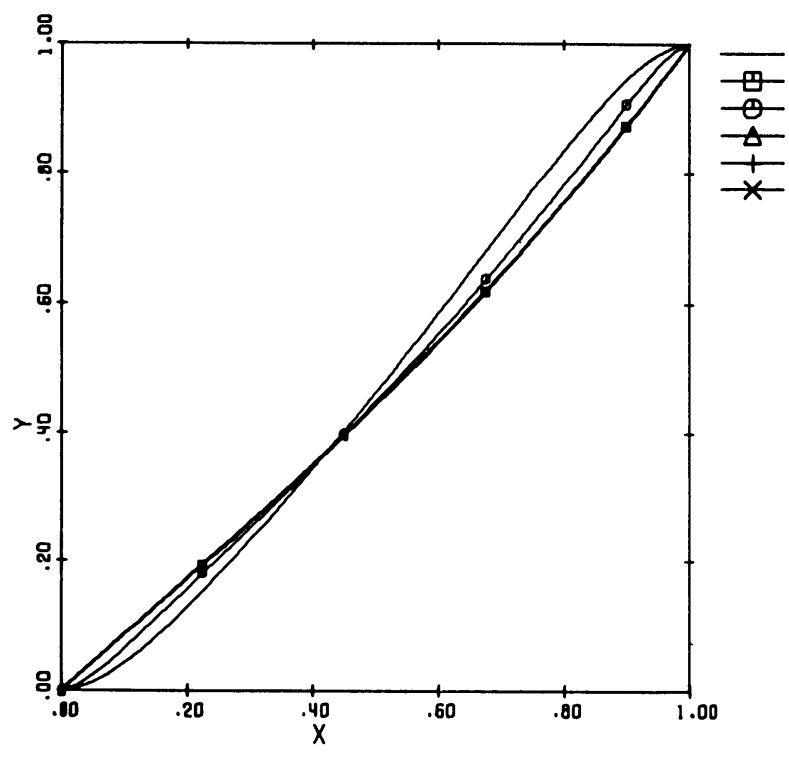

EXACT SOLN. EPS $=0.01$

ASYM. SOLN. EPS $=0.01$

EXACT SOLN. EPS $=0.001$

ASYM. SOLN. EPS $=0.001$

ASYM. SOLN. EPS $=0.0001$

ASYM. SOLN. EPS $=0.00001$

Figure 7

Exact and asymptotic solutions of Example 4: $\epsilon y^{i v}-(1+\epsilon) y^{\prime \prime}+y=0$,

$$
y(0)=y^{\prime}(0)=y^{\prime}(1)=0, y(1)=1
$$

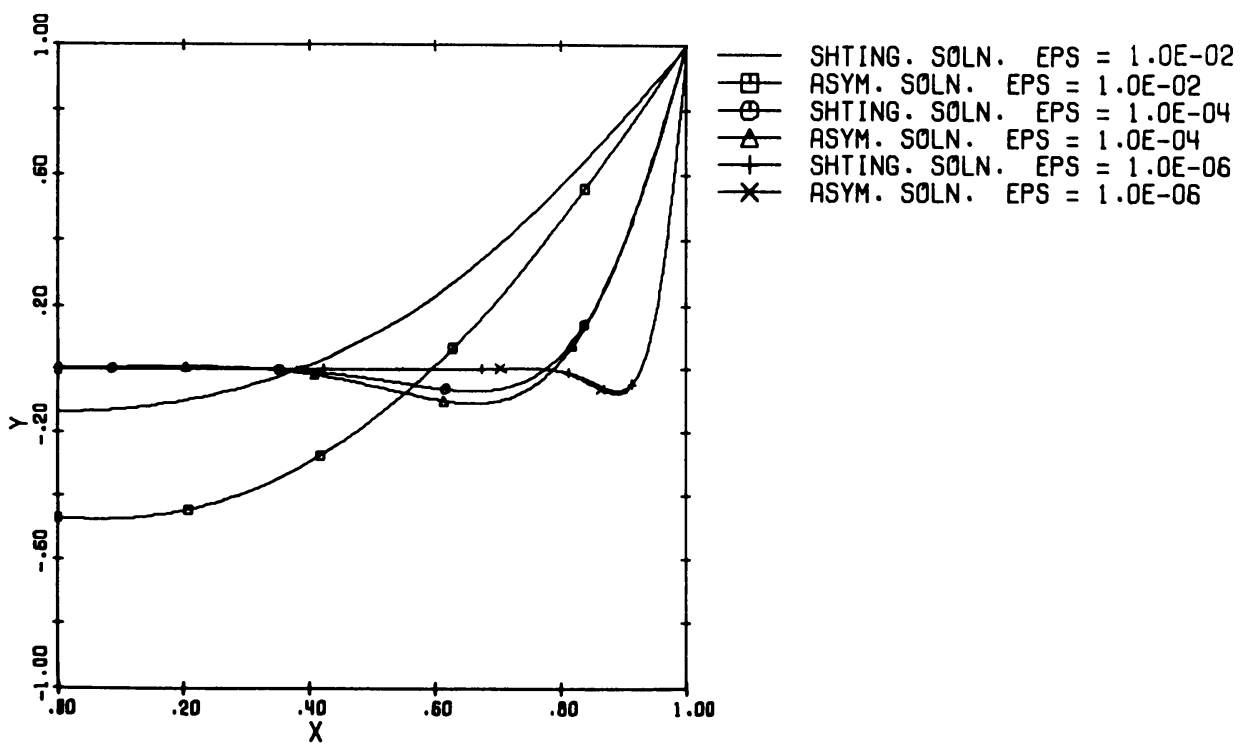

Figure 8

Asymptotic and shooting solutions of Example 5: $\epsilon\left(e^{\lambda x^{6}} y^{\prime \prime}\right)^{\prime \prime}+y=0$,

$$
y^{\prime}(0)=y^{\prime \prime \prime}(0)=y^{\prime \prime}(1)=0, y(1)=1
$$




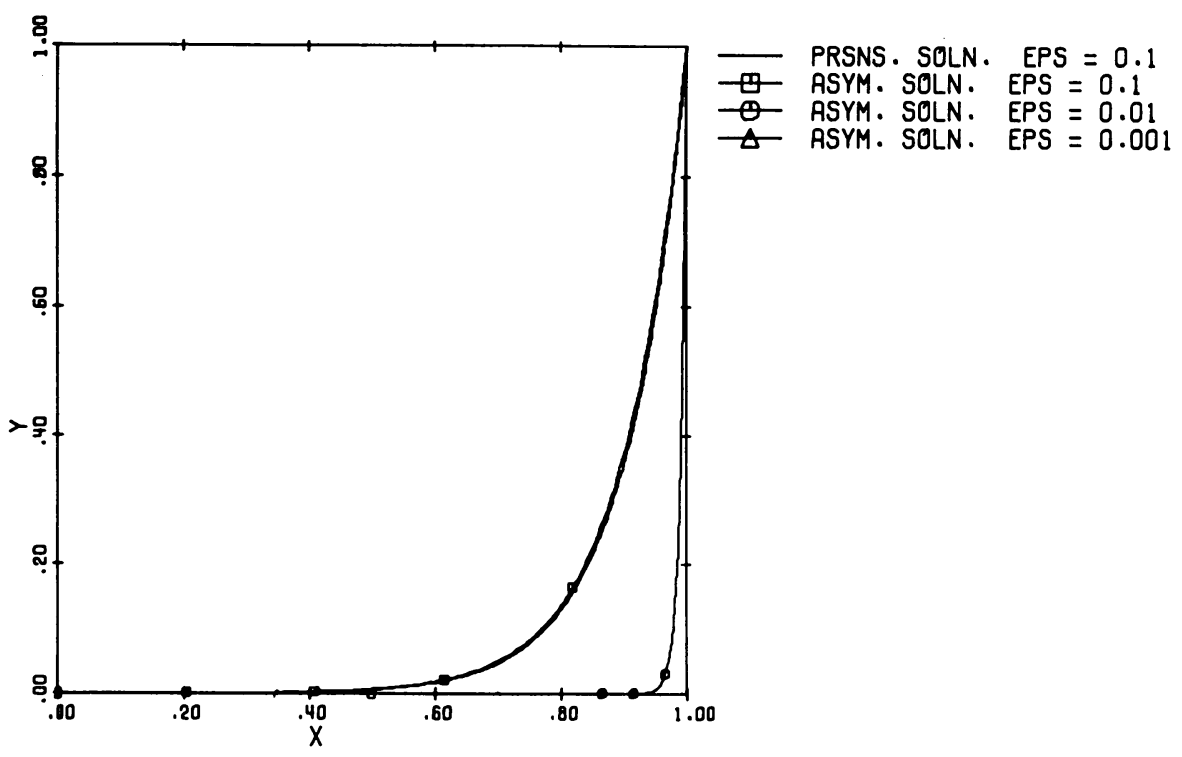

Figure 9

Asymptotic and Pearson's method solutions of Example 6: $\epsilon y^{\prime \prime}-$ $y^{\prime}-y^{2}=0, y^{\prime}(0)+y(0)=0, y(1)=1$. The solution of the reduced problem sought is $z(x)=0$

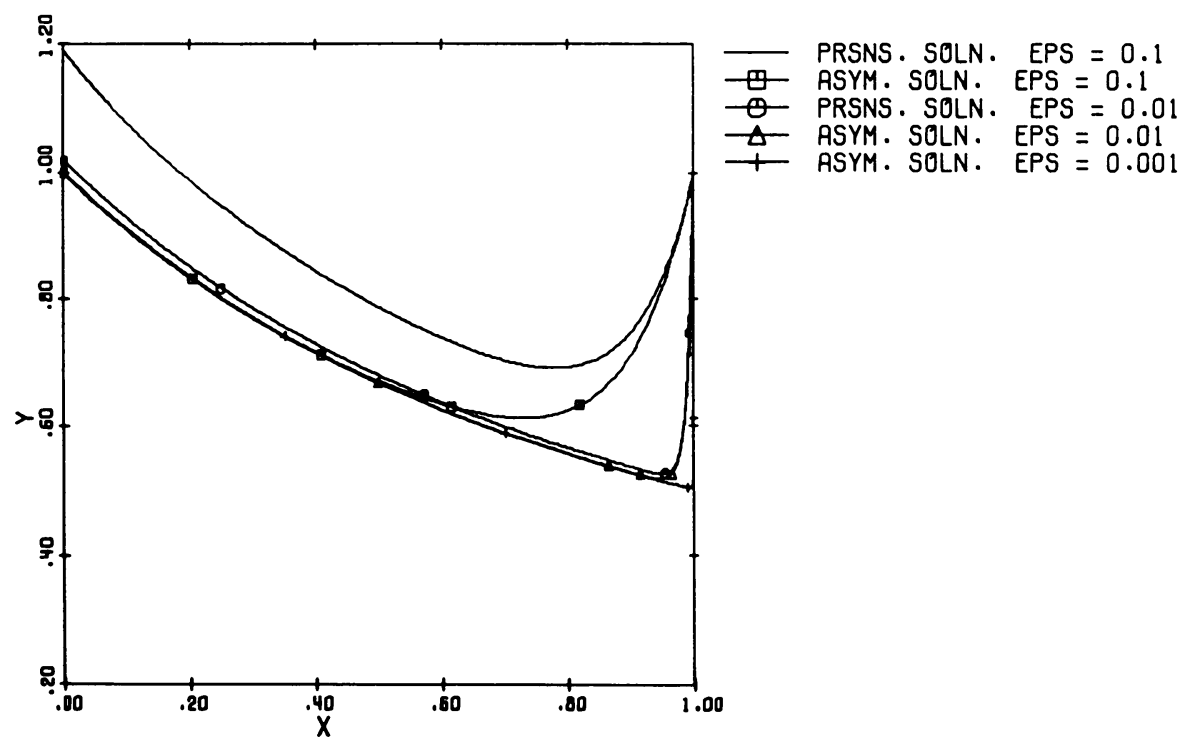

Figure 10

Asymptotic and Pearson's method solutions of Example 6: $\epsilon y^{\prime \prime}-$ $y^{\prime}-y^{2}=0, y^{\prime}(0)+y(0)=0, y(1)=1$. The solution of the reduced problem sought is $z(x)=(1+x)^{-1}$ 


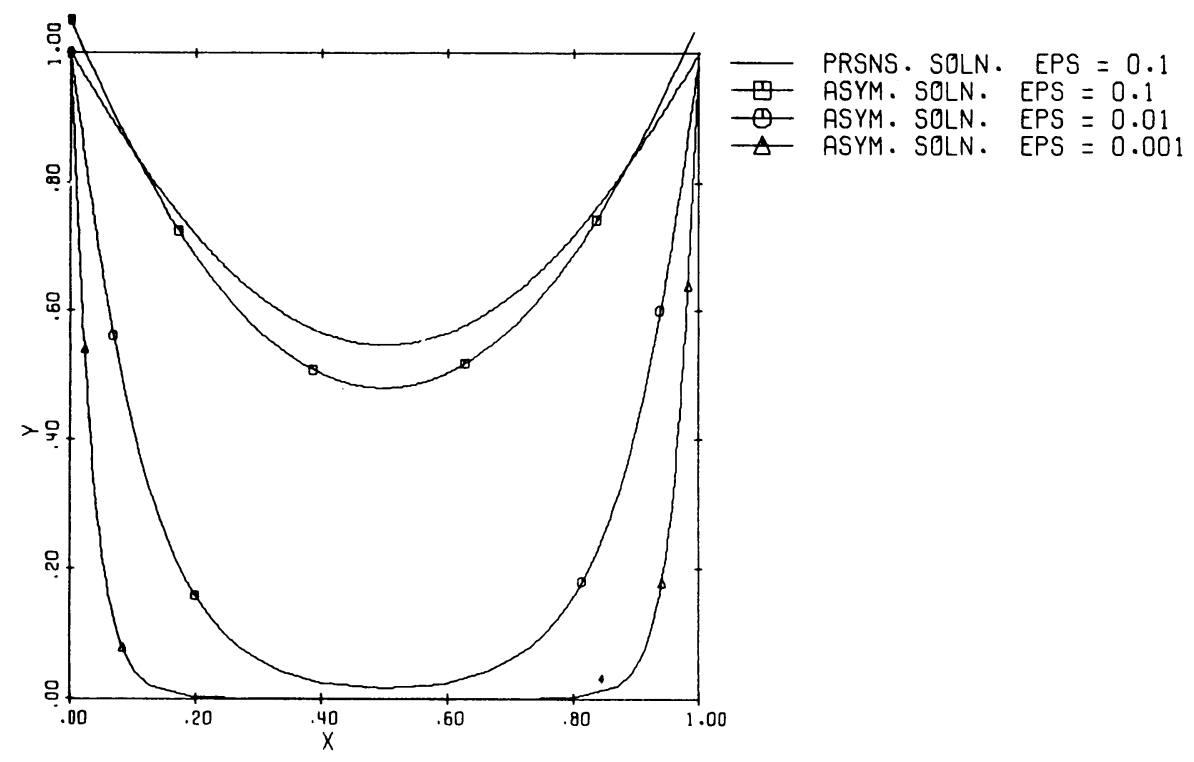

FIgURE 11

Asymptotic and Pearson's method solutions of Example 7:

$$
\epsilon y^{\prime \prime}-y+y^{3}=0, y(0)=y(1)=1
$$

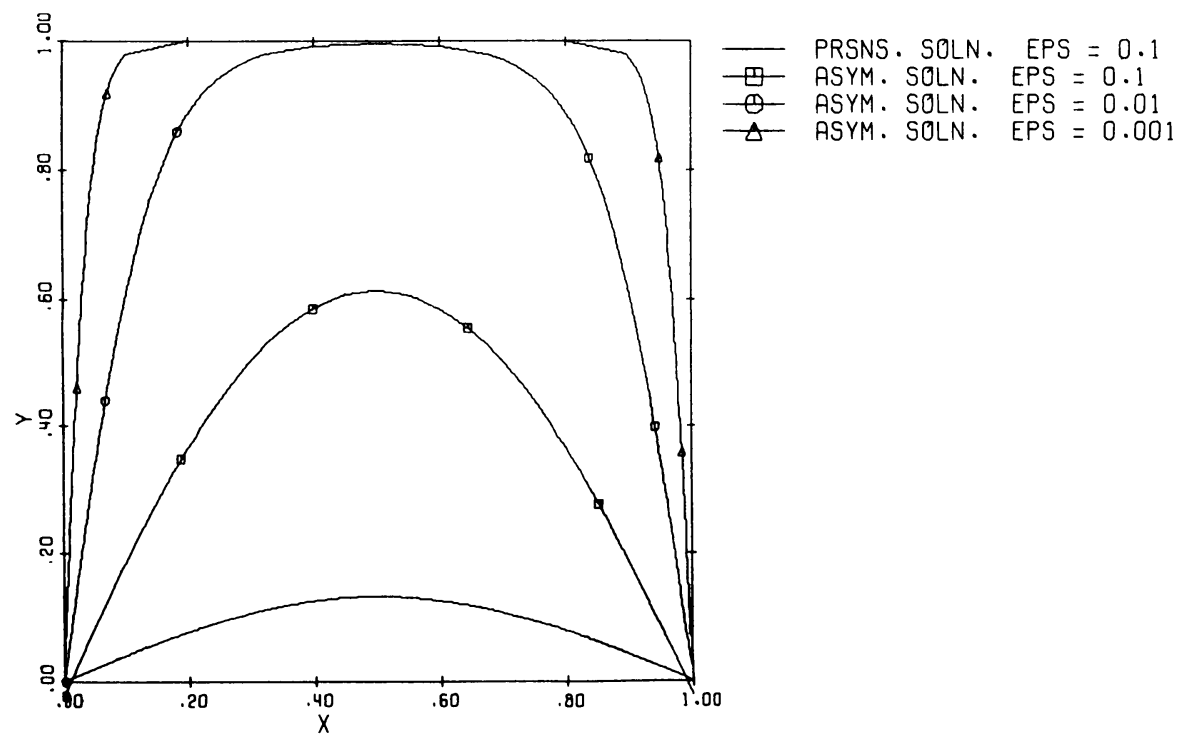

Figure 12

Asymptotic and Pearson's method solutions of Example 8:

$$
\epsilon y^{\prime \prime}+y-y^{3}=0, y(0)=y(1)=0
$$


Example 5. $\epsilon\left(e^{\lambda x^{6}} y^{\prime \prime}\right)^{\prime \prime}+y=0, y^{\prime}(0)=y^{\prime \prime \prime}(0)=y^{\prime \prime}(1)=0, y(1)=1$. This equation is a modification of one described by Timoshenko [39]. Physically, $1-y$ is proportional to the deflection of an elastically weak (or long), uniformly loaded, simply supported, variable thickness beam on an elastic foundation. The thickness of the beam is $t_{0} e^{\lambda^{1 / 3} x^{2}}$. Runs were made for $\epsilon=10^{-i} ; i=2,4,6$, and comparisons between asymptotic and shooting solutions are presented in Table 5 and Figure 8 . The solution $y$ has a weak boundary layer of thickness $O\left(\epsilon^{1 / 4}\right)$ at $x=1$. The slow decay of the boundary layer accounts for our rather poor agreement for $\epsilon \geqslant 10^{-4}$.

Example 6. $\epsilon^{2} y^{\prime \prime}-y^{\prime}-y^{2}=0, y^{\prime}(0)+y(0)=0, y(1)=1$. Since the nonuniform convergence occurs at $x=1$ the reduced problem

$$
z^{\prime}+z^{2}=0, \quad z^{\prime}(0)+z(0)=0
$$

has two solutions $z(x)=0$ and $z(x)=(1+x)^{-1}$. Corresponding multiple solutions of the full problem result (cf. O'Malley [30]). Results obtained by the asymptotic method and by Pearson's method are compared for both solutions in Table 6 for $\epsilon=10^{-i}$, $i=1, \ldots, 5$. Graphs of some of these results are presented in Figures 9 and 10 for the solutions corresponding to $z(x)=0$ and $z(x)=(1+x)^{-1}$, respectively.

Example 7. $\epsilon y^{\prime \prime}-y+y^{3}=0, y(0)=y(1)=1$. The limiting solution $z(x)=0$ satisfies all of our hypotheses for these boundary conditions and requires a boundary layer at both endpoints. It is interesting to note that these boundary conditions also allow the solution $y(x)=1$ even though the limiting solutions $z(x)= \pm 1$ do not satisfy our restrictions. (In our defense, however, we note that the "nearby" problem given by the same differential equation with $y(0)=y(1)=0.99$ would not have a solution tending to 1.) Runs were made for $\epsilon=10^{-i}, i=1, \ldots, 5$, and comparisons between solutions obtained by the asymptotic method and by Pearson's method for the limiting solution $z(x)=0$ are presented in the first three columns of Table 7 and in Figure 11.

Example 8. $\epsilon y^{\prime \prime}+y-y^{3}=0, y(0)=y(1)=0$. The limiting solutions $z(x)=$ \pm 1 follow under our hypotheses for these boundary conditions. While the trivial solution $y(x)=0$ does not satisfy our hypotheses, it is also valid for these boundary conditions. In addition, O'Malley [31] shows that there are denumerably many solutions of this problem switching back and forth between \pm 1 . The results of calculations corresponding to the limiting solution $z(x)=1$ obtained by the asymptotic method and Pearson's method are presented in the last two columns of Table 7 and in Figure 12. Although exact solutions of these last problems could be obtained using elliptic integrals, we have not done so.

5. Conclusions. The results of the previous section indicate that our procedure can be used to obtain accurate numerical solutions of very stiff ordinary differential equations with very little computational effort. The accuracy of our methods depends on the magnitude of the small coefficients in the equation as well as the amount by which the coefficients vary and the thickness of the boundary layers. For example, the results of Section 4 clearly indicate that our methods are accurate even when the magnitude of the small parameters, say $\epsilon$, is moderate in size provided that the boundary layers are of thickness $O(\epsilon)$ or $O(\sqrt{\epsilon})$. However, in Example 5, where the bound- 
ary layer is of thickness $O\left(\epsilon^{1 / 4}\right)$ our results become accurate only for $\epsilon \leqslant 10^{-6}$. Since our results are asymptotic as the stiffness increases, they should not be used for slightly stiff, and should be used cautiously for moderately stiff equations.

We envision that asymptotic methods like ours could form part of a computational library of methods for solving ordinary differential equations. Such a library would contain a general purpose method, like, for example, Keller's adaptive grid procedures [17], [18], [20] which would be used for the majority of problems, while an asymptotic method would be used for very stiff problems. In addition, our results could provide an initial approximation to an adaptive grid procedure for slightly stiff or moderately stiff problems (cf. Yarmish [45], [46]).

We anticipate that combined asymptotic and numerical methods could be developed for more complicated equations, e.g., turning point problems and higher order nonlinear systems. We hope that this investigation might prove useful in developing further results.

Acknowledgement. We wish to thank Joseph B. Keller for his encouragement and support of this effort, Bernard Matkowsky for his suggestions, and Rebecca A. Kiesman for her help with some of the programming.

Department of Mathematical Sciences

Rensselaer Polytechnic Institute

Troy, New York 12181

Department of Mathematics

University of Arizona

Tucson, Arizona 85721

1. L. R. ABRAHAMSSON, H. B. KELLER \& H. O. KREISS, "Difference approximations for singular perturbations of systems of ordinary differential equations," Numer. Math., v. 22, 1974, pp. 367-391.

2. R. AIKEN \& L. LAPIDUS, "An effective numerical integration method for typical stiff systems," A.I.Ch.E.J., v. 20, 1974, pp. 368-375.

3. P. T. BOGGS, "A minimization algorithm based on singular perturbation theory," SIAM J. Numer. Anal. (To appear.)

4. R. BULIRSCH \& J. STOER, "Numerical treatment of ordinary differential equations by extrapolation methods," Numer. Math., v. 8, 1966, pp. 1-13. MR 32 \#8504.

5. J. D. COLE, Perturbation Methods in Applied Mathematics, Blaisdell, Waltham, Mass., 1968. MR 39 \#7841.

6. S. D. CONTE, "The numerical solution of linear boundary value problems," SIAM Rev., v. 8, 1966, pp. 309-321. MR $34 \# 3792$.

7. S. D. CONTE \& C. de BOOR, Elementary Numerical Analysis, 2nd ed., McGraw-Hill, New York, 1972, Chapter 5.

8. F. W. DORR, "The numerical solution of singular perturbations of boundary value problems," SIAM J. Numer. Anal., v. 7, 1970, pp. 281-313. MR 42 \#2683.

9. F. W. DORR, "An example of ill-conditioning in the numerical solution of singular perturbation problems," Math. Comp., v. 25, 1971, pp. 271-283. MR 45 \#6200.

10. W. E. FERGUSON, JR., "A singularly perturbed linear two-point boundary-value problem,” Ph.D. Dissertation, California Inst. Tech., 1975.

11. P. C. FIFE, "Semilinear elliptic boundary value problems with small parameters," Arch. Rational Mech. Anal., v. 52, 1973, pp. 205-232. MR 51 \#10863.

12. P. C. FIFE, "Transition layers in singular perturbation problems," J. Differential Equations, v. 15, 1974, pp. 77-105. MR 48 \#002.

13. N. FRÖMAN \& P. O. FRÖMAN, JWKB Approximation. Contributions to the Theory, North-Holland, Amsterdam, 1965. MR 30 \#3694. 
14. C. W. GEAR, Numerical Initial Value Problems in Ordinary Differential Equations, Prentice-Hall, Englewood Cliffs, N. J., 1971, Chapters 9, 11. MR 47 \#4447.

15. P. W. HEM KER, A Method of Weighted One-Sided Differences for Stiff Boundary Value Problems with Turning Points, Report NW 9/74, Mathematisch Centrum, Amsterdam, 1974. MR 50 \#3587.

16. F. A. HOWES, "The asymptotic solution of a class of singularly perturbed nonlinear second order boundary value problems via differential inequalities," SIAM J. Math. Anal. (To appear.)

17. H. B. KELLER, "Accurate difference methods for linear ordinary differential systems subject to linear constraints," SIAM J. Numer. Anal., v. 6, 1969, pp. 8-30. MR 40 \#6776.

18. H. B. KELLER, "Accurate difference methods for nonlinear two-point boundary value problems," SIAM J. Numer. Anal., v. 11, 1974, pp. 305-320. MR 50 \#3589.

19. H. B. KELLER \& T. CEBECI, "Accurate numerical methods for boundary-layer flows. II. Two-dimensional turbulent flows," $A I A A$ J., v. 10, 1972, pp. 1193-1199. MR 46 \#10300.

20. H. B. KELLER \& A. B. WHITE, JR., "Difference methods for boundary value problems in ordinary differential equations," SIAM J. Numer. Anal., v. 12, 1975, pp. 791-802.

21. M. LENTINI \& V. PEREYRA, "Boundary problem solvers for first order systems based on deferred corrections," in Numerical Solution of Boundary Value Problems for Ordinary Differential Equations, (A. K. Aziz, Editor), Academic Press, New York, 1975.

22. B. LINDBERG, "On a dangerous property of methods for stiff differential equations," $B I T$, v. 14, 1974, pp. 430-436. MR 50 \#15347.

23. D. B. MACMILLAN, "Asymptotic methods for systems of differential equations in which some variables have very short response times," SIAM J. Appl. Math., v. 16, 1968, pp. 704-722. MR 38 \#2403.

24. J. A. M. McHUGH, "An historical survey of ordinary differential equations with a large parameter and turning points," Arch. History Exact Sci., v. 7, 1971, pp. 277-324.

25. W. L. MIRANKER, "Numerical methods of boundary layer type for stiff systems of differential equations," Computing, v. 11, 1973, pp. 221-234.

26. W. L. MIRANKER \& J. P. MORREEUW, "Semianalytic studies of turning points arising in stiff boundary value problems," Math. Comp., v. 28, 1974, pp. 1017-1034.

27. D. E. MULLER, "A method for solving algebraic equations using an automatic computer," MTAC, v. 10, 1956, pp. 208-215. MR 18, 766.

28. W. D. MURPHY, "Numerical analysis of boundary-layer problems in ordinary differential equations," Math. Comp., v. 21, 1967, pp. 583-596. MR 37 \#1089.

York, 1974

30. R. E. O'MALLEY, JR., "On multiple solutions of a singular perturbation problem," Arch. Rational Mech. Anal., v. 49, 1972/73, pp. 89-98. MR 49 \#761.

31. R. E. O'MALLEY, JR., "Phase plane solutions to some singular perturbation problems," J. Math. Anal. Appl, v. 54, 1976, pp. 449-466.

32. R. E. O'MALLEY, JR., "Boundary layer methods for ordinary differential equations with small coefficients multiplying the highest derivatives," (Proc. Sympos. on Constructive and Computational Methods for Differential and Integral Equations), Lecture Notes in Math., vol. 430, Springer-Verlag, Berlin and New York, 1974, pp. 363-389.

33. R. E. O'MALLEY, JR. \& J. B. KELLER, "Loss of boundary conditions in the asymptotic solution of linear ordinary differential equations. II. Boundary value problems," Comm. Pure Appl. Math., v. 21, 1968, pp. 263-270. MR 37 \#528.

34. F. W. J. OLVER, Asymptotics and Special Functions, Academic Press, New York, 1974. published.)

36. C. E. PEARSON, "On a differential equation of the boundary layer type," J. Math. and Phys., v. 47, 1968, pp. 134-154. MR 37 \#3773.

37. C. E. PEARSON, "On non-linear ordinary differential equations of boundary layer type," J. Math. and Phys., v. 47, 1968, pp. 351-358. MR 38 \#5400.

38. M. R. SCOTT \& H. A. WATTS, Support-A Computer Code for Two-Point BoundaryValue Problems via Orthonormalization, Sandia Laboratories Report SAND 75-0198, June 1975.

39. S. TIMOSHENKO, Strength of Materials, Part II, Advanced Theory and Problems, 3rd ed., Van Nostrand, Princeton, N. J., 1956.

40. M. I. VIŠIK \& L. A. LJUSTERNIK, "Initial jump for non-linear differential equations containing a small parameter," Dokl. Akad. Nauk SSSR, Tom 132, 1960, pp. 1242-1245= Soviet Math. Dokl., v. 1, 1960, pp. 749-752. MR 22 \#11181. 
41. W. R. WASOW, "On the asymptotic solution of boundary value problems for ordinary differential equations containing a parameter," J. Math. and Phys., v. 23, 1944, pp. 173-183. MR $6,86$.

42. W. R. WASOW, "Singular perturbations of boundary value problems for nonlinear differential equations of the second order," Comm. Pure. Appl. Math., v. 9, 1956, pp. 93-113. MR $18,39$.

43. W. R. WASOW, "Connection problems for asymptotic series," Bull. Amer. Math. Soc., v. 74, 1968, pp. 831-853. MR 37 \#336.

44. R. WILlOUGHBY (Editor), Stiff Differential Systems, Plenum Press, New York, 1974.

45. J. YARMISH, Aspects of the Numerical and Theoretical Treatment of Singular Perturbation, Doctoral Dissertation, New York Univ., 1972.

46. J. YARMISH, "Newton's method techniques for singular perturbations," SIAM J. Math. Anal., v. 6, 1975, pp. 661-680. 